\title{
Biomass-fired combined heating, cooling, and power for small scale applications - A review
}

\author{
Moritz Wegenera,b,*, Anders Malmquista, Antonio Isalguéb, Andrew Martina \\ a Heat and Power Technology, Department of Energy Technology, KTH Royal Institute of Technology, \\ Brinellvägen 68, 10044 Stockholm, Sweden \\ bArchitecture \& Energy, School of Architecture of Barcelona, UPC. Av. Diagonal, 649, 6th Floor, 08028 \\ Barcelona, Spain \\ ${ }^{*}$ Corresponding Author: \\ Tel.: +4687906000 \\ E-mail: mbgw@kth.se
}

Declaration of interest: None

\begin{abstract}
The growing demand for energy and the accelerating threats from climate change call for innovative and sustainable solutions to decrease dependency on fossil fuels. Biomass-based, small-scale Combined Cooling, Heating and Power (CCHP) systems are one of these solutions, because they can satisfy the energy demands of the consumer with enhanced flexibility, lower losses, less costs and less environmental pollution as compared to centralized facilities. Due to recent advances in several scientific subfields with relevance to small-scale CCHP, a rapidly increasing amount of literature is now available. Therefore, a structural overview is essential for engineers and researchers. This paper presents a review of the current investigations in small-scale CCHP systems covering biomass-fired concepts and solar extensions. To this end, critical system components are described and analysed according to their specific advantages and drawbacks. Recent case studies have been collected and key findings are highlighted according to each type of prime mover. The results indicate a scientific bias towards the economic viability of such systems and the need for real-life and experiment system data. However, the potential of biomass-fired CCHP systems and of such systems with solar extensions has clearly been recognised. Based on the results, future policy implementations should focus on fostering such systems in areas with high energy costs and to increase energy resilience in developed regions. Additionally research and industry applying novel prime mover technologies should be financially supported.
\end{abstract}

\section{Keywords}

Small-scale CCHP, Trigeneration, biomass, bio-solar

\section{Introduction}

To fuel the world's rising demand for energy while also to slow down emissions of greenhouse gases, more efficient and more sustainable energy systems are necessary [1]. Combined Cooling, Heating and Power (CCHP) is a technology that aims to raise the energy efficiency of an energy system by using the electric as well as the thermal output for practical applications [2,3]. Most deployed CCHP systems involve centralized facilities with extensive heating and cooling networks supplying hundreds or thousands of industrial and residential consumers. However, the disadvantages of centralized energy systems are losses due to longer transfer distances, the inability to reply to the immediate demands of individual consumers and higher security risks should unexpected shutdowns occur [4]. Therefore smaller decentralized units, which serve the local demand for heat as well as for electricity, can be a more profitable and more efficient alternative to centralized facilities [5]. 
While small-scale systems providing cooling, heat and/ or power driven by fossil fuels haven been successfully developed and constructed for decades, the implementation of renewable options is relatively new and successful mass-scale commercialization still has to be proven [6,7]. Amongst renewable energies, biomass seems to be the most promising energy source for CCHP systems as other renewable energies are either not generating enough heat in normal operation modes (wind, photovoltaic, hydro), are too locally limited (geothermal) or are too volatile (solar thermal) [8- 11]. Hence, the development of small-scale, biomass-fired CCHP systems is becoming increasingly important for climate politics, economics and research [12- 14]. Such systems can be especially costefficient solutions in remote areas and islands with adequate sources of renewable energies $[15,16]$. Extensions involving solar electric energy can greatly augment the sustainability and viability of this approach, considering the vast solar potential and falling photovoltaic (PV) prices [17].

Historically CCHP is linked to the more well-known Combined Heat and Power (CHP) concept: a cooling unit is integrated to the CHP system, leading to more choices for energy outputs and higher operating times, especially in comparison to conventional power plants [12,18]. Several synonyms of CCHP can be found in literature and the following list defines their meaning for this paper [19]:

- Trigeneration: essentially equivalent to CCHP [19]

- Polygeneration/Multigeneration: Any system which produces more than two energy services; this can be a CCHP system but may also be a system producing chemicals or other products [19,]

- MCCHP/ $\mu$ CCHP: Micro CCHP (with less than $20 \mathrm{~kW}$ electric power) $[20,21]$

- CHP with Cooling or Cogeneration with Cooling: Essentially the same as CCHP [22]

- BCHP: Building Cooling Heating and Power [23]

- Biorefinery: Any system, which produces chemical products out of biomass [19]

Several studies indicate significant potential for biomass-fired CCHP systems, but so far only mediumscale (1 MW-10 MW) and large scale (>10 MW) systems have been commercialised successfully, while micro-scale $(<20 \mathrm{~kW})$ and small-scale $(20 \mathrm{~kW}-1 \mathrm{MW})$ systems are still in an experimental phase $[21,24]$. To design a CCHP system for optimal environmental and economic performance, component type and size have to be evaluated and chosen carefully according to the electricity, heating and cooling demand [2,5]. For this, many authors argue that the prime mover is the heart of such systems with the biggest impact on system economics and environment [5,21,25]. Another possibility to optimize smallscale CCHP systems is to use operation strategies adjusted to the electric or the thermal load [20]. These loads depend heavily on the energy consumer, which can be a household, an office building, a hospital, or any similar buildings [26]. Several studies investigate the optimal behaviour for CCHP systems and highlight the benefits of their flexibility for energy generation $[19,27,28]$.

The previously mentioned studies review certain aspects of CCHP systems, but none of them focus on the growing amount of literature on biomass-fired options [14]. Hence, the aim of this paper is to summarize and structure the constantly increasing amount of scientific literature on small-scale $(<1$ $\mathrm{MW}_{\mathrm{el}}$ ), biomass-fired CCHP systems. For this, currently used technologies are presented as shown in Figure 1 and their specific benefits as well as drawbacks are identified. For each different prime mover technology, the key findings of the most recent case studies from 2010 to 2017 are highlighted and their key characteristics (maximal output, type of biomass, prime mover type, refrigeration technology, model software/ experiment location) are assembled. 


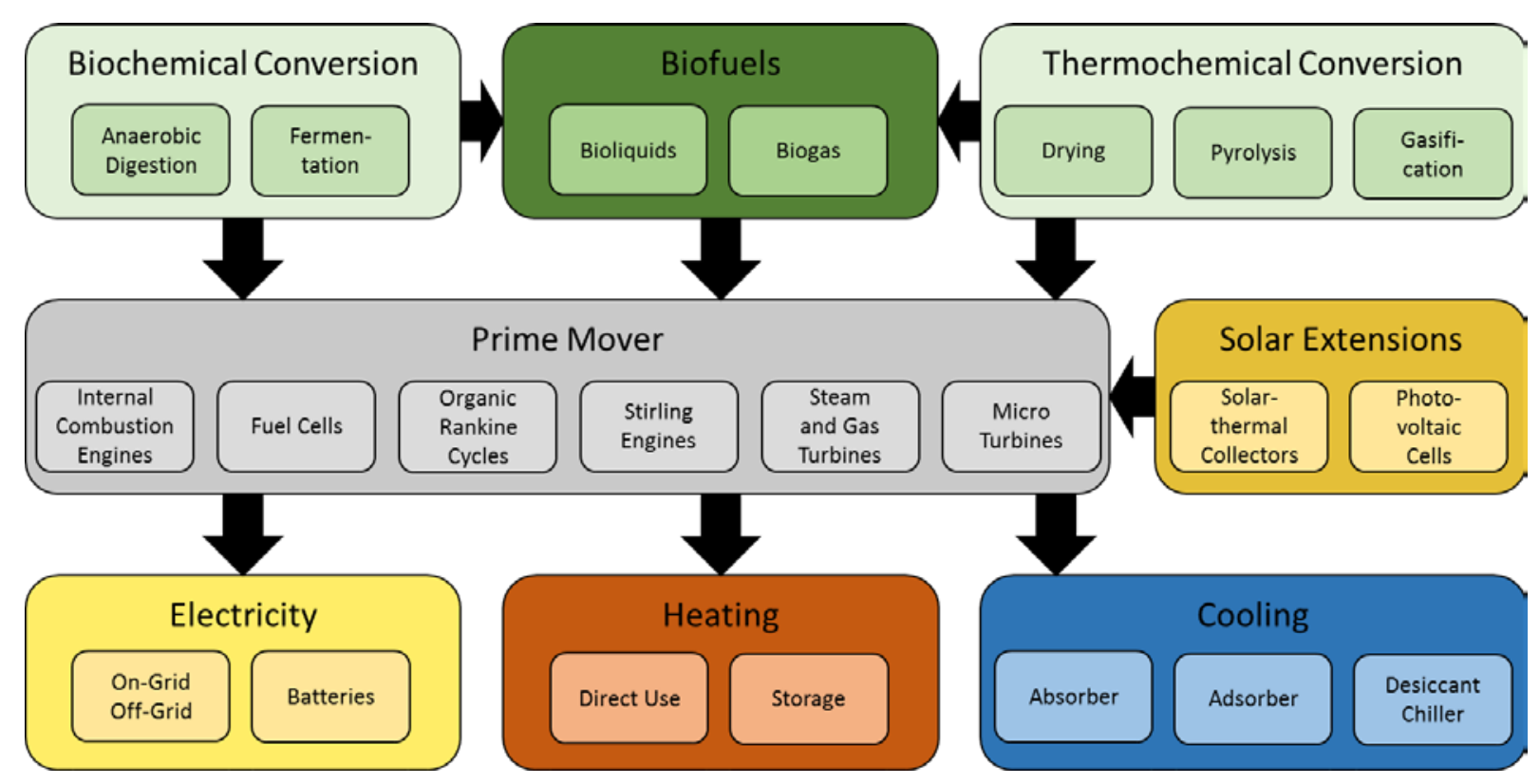

Figure 1 Key components in biomass-fired and solar assisted small-scale CCHP systems

After this introduction, internal and external factors that affect the technological and economic development of CCHP systems will be listed in chapter 2 . In chapter 3 , the different ways to obtain biofuel for small-scale CCHP systems are presented. The studies investigating small-scale biomassfired CCHP systems are summarized and listed according to their prime mover in chapter 4 . Therefore, the focus of the review has been laid on CCHP systems based on these various prime movers. Chapter 5 presents the most common cooling and storage technologies. The findings are discussed in chapter 6 and finally conclusions are drawn in chapter 7, where also future policy implementations are suggested.

\section{Internal and external factors driving CCHP development}

To seize the potential of small-scale CCHP systems internal as well as external factors will influence the development in the coming years. One external factor for the viability of small-scale CHP and CCHP systems are government policies for support and subsidies [19]. It was noted that governments from developed and developing countries like the USA, China, the EU, Brazil, Russia and J apan use different mechanisms to promote the use of CCHP systems $[20,21]$. This implies, that the huge potential has been acknowledged also politically, but until today the total share of electricity and heat generated through CHP and CCHP systems still remains low with for example less than $6 \%$ of heat generated by CHP or CCHP systems in the EU in 2016 [20,29]. However, other external factors like biomass prices, oil prices or carbon dioxide taxes also effect the economics of small-scale CCHP systems greatly [30].

There are several parameters to measure the performance of internal factors of energy systems, for which a good overview can be found in [31]. One of the most commonly used energetic performance parameters is the Primary Energy Saving Ratio (PESR), which describes the ratio of primary energy saved in the new system compared to a conventional stand-alone energy generation system [12,31]. Many small-scale CHP and CCHP systems achieve a PESR of 20-30\%, which indicates favourable ecologic and economic performances [9,27,32]. However, especially in the case of biomass-fired and biomass-fired solar-assisted CCHP systems, the PESR can be misleading, because even if more energy is needed in biomass systems than in conventional systems, they might still perform ecologically better. Hence, other methodologies like Life Cycle Assessments or $\mathrm{CO}_{2}$-equivalence should also be taken into account [2,31].

\section{Fuel Supply}

Apart from using simple mechanical preparation, biomass can be transformed into a gaseous, liquid or solid fuel through either biochemical conversion or through thermochemical conversion [33]. These 
products can either be used for fueling a CCHP system directly or can be stored and then transported to the CCHP system.

\subsection{Biochemical Conversion}

The most popular biochemical conversion technology is anaerobic digestion, which describes a chain process of biological reactions in a low-oxygen environment transforming organic biomass into biogas $[10,34]$. The main contents of biogas are methane with 55-80\% and carbon dioxide with $20-45 \%$, while other gases as impurities and the remains of the anaerobic biomass are also produced [33,35]. Biomass used for anaerobic digestion is usually waste from agricultural enterprises or manure from animals and/ or humans. This type of biomass has usually a high moisture content and features high ash contents [36].

Another technology for the biochemical conversion of biomass is fermentation, which describes the conversion of biomass firstly to sugars and finally to alcohols, mainly bio-ethanol. Mostly sugar or starch crops are used, however the process can also be applied to lignocellulose biomass, but with less efficiency [36]. Fermentation is usually applied in large-scale factories and is one of the most commonly used processes for the production of high-quality biofuels [37].

\subsection{Thermochemical Conversion}

The first process for preparing biomass for thermochemical conversion is drying, because this way, even for direct combustion, the efficiency can easily be increased by 5-10\% and air emissions can be lowered. For micro-scale CCHP systems, the biomass can be dried before insertion into the system by using direct solar irradiation or other heat sources. Alternatively, some part of the exhaust heat from the CCHP system can be used to dry the biomass rapidly [38].

Pyrolysis describes the process of breaking down long hydrocarbon chains into smaller pieces by applying heat at temperatures, normally between $450-600^{\circ} \mathrm{C}$ with low-oxygen supply [36]. Apart from temperature and heat supply time, other factors like chemical composition or surface area of the biomass have significant impact on the process and the products [35,39]. Gaseous products of biomass pyrolysis are mostly $\mathrm{H}_{2}, \mathrm{CO}, \mathrm{CO}_{2}$, and $\mathrm{CH}_{4}$, but also higher hydrocarbons like $\mathrm{C}_{2} \mathrm{H}_{6}, \mathrm{C}_{2} \mathrm{H}_{4}$, and $\mathrm{C}_{3} \mathrm{H}_{8}$ in small amounts. Additionally liquid oils are produced, which consist of even higher hydrocarbon chains, and solid charcoal remains, which can be used for combustion or fertilizing [39]. The produced oils can be transformed into transportation fuels or they can be further broken down by applying more heat $[10,35]$.

This process of applying even more heat to biomass, usually at temperatures between $750-1,000{ }^{\circ} \mathrm{C}$ degrees, is referred to as gasification. At this temperature level, almost all carbohydrates are cracked down to $\mathrm{H}_{2}$ and $\mathrm{CO}$ and the produced gas is often referred to as synthesis gas (syngas), producer gas, or wood gas. This is not a full combustion because the process occurs in a low oxygen environment [35]. Fixed bed type gasifiers represent the simplest category of gasifiers, where solid fuel particles are placed in a cylinder and gasifying agents (e.g. air, oxygen, steam) pass through the solid particles. After leaving the gasification chamber, the syngas usually undergoes a cleaning process by sending it through a cyclone for removal of solid particles and through filters. Another category of gasifiers is fluidized bed gasifiers [40]. Here the fluidizing agents enter from below into a mixture of biomass and inert bed materials like silica or sand, which are working as catalysts for the gasification process.

\subsection{Bioliquids and Biogases}

Based on the previous processes, medium to large-scale biofuel plants can provide biofuels used in many small-scale CCHP systems. For the sake of a complete picture of possible biofuel sources, a few other processes for the generation of biofuels will be listed shortly:

- J atropha oil produced mechanically from J atropha plants holds high potential for an easy-toproduce supply of biodiesel [41]. Several other so called energy plants are also currently under investigation for efficient generation of biofuels, e.g. Switch grass, Bermuda grass, Silver grass, Alamo [42]. 
- For the production of methane, seaweed (macroalgae) is currently being investigated and several pilot projects have proven successful implementation for biofuel production [42].

- Microalgae, which are used mainly in urban wastewaters, have been shown to be feasible for biofuel production [43].

\section{Prime Mover}

For the various types of biofuels, different prime movers can be selected, which greatly influence the energetic and economic performance of a CCHP plant [21,25,44]. An assessment of key characteristics of prime movers presented in this study is shown in Table 1 (No publications could be found that considered steam engines in this context, thus this prime mover is omitted). The assessment of the potential for a given prime mover estimates the ability for biomass-fired CCHP market penetration in the near future. It is based on findings from different sources as well as on the authors' judgement.

Table 1 Assessment of Prime Movers for small-scale biomass-fired CCHP systems

\begin{tabular}{|c|c|c|c|c|c|}
\hline Prime Mover & $\begin{array}{c}\text { Internal } \\
\text { Combustion } \\
\text { Engines }\end{array}$ & $\begin{array}{l}\text { Fuel } \\
\text { Cells }\end{array}$ & $\begin{array}{l}\text { Stirling } \\
\text { Engines }\end{array}$ & $\begin{array}{l}\text { Organic } \\
\text { Rankine } \\
\text { Cycles }\end{array}$ & $\begin{array}{l}\text { Micro } \\
\text { Turbines }\end{array}$ \\
\hline Size & $\begin{array}{c}\text { Up to near } \\
100 \mathrm{MW}[25]\end{array}$ & Up to $2 M W$ [25] & Up to $150 \mathrm{~kW}$ [2] & up to $2 M W[2]$ & $\begin{array}{c}\text { Up to } 1,000 \mathrm{~kW} \\
{[21]}\end{array}$ \\
\hline Advantages & $\begin{array}{c}\text { Very high reliability } \\
\text { (with clean fuels) } \\
\qquad[21,45] \\
\text { Rapid start-up [21] } \\
\text { Low investment } \\
\text { costs [21] }\end{array}$ & $\begin{array}{c}\text { Excellent partial } \\
\text { load } \\
\text { performance } \\
{[25]} \\
\text { Very high } \\
\text { electric } \\
\text { efficiency }[5,21] \\
\text { Low noise }[45] \\
\text { Low emissions } \\
{[5,21]}\end{array}$ & $\begin{array}{c}\text { Can use low } \\
\text { quality fuels due to } \\
\text { external } \\
\text { combustion } \\
{[21,25,32]} \\
\text { Good partial load } \\
\text { performance } \\
{[12,25,32]} \\
\text { Potentially low } \\
\text { maintenance } \\
\text { requirements/ less } \\
\text { moving parts } \\
{[21,46]} \\
\text { High thermal } \\
\text { efficiency [5] } \\
\text { Low emissions } \\
{[5,32]}\end{array}$ & $\begin{array}{c}\text { Can run with low } \\
\text { grade heat [12] } \\
\text { High reliability } \\
{[12,47]} \\
\text { Low maintenance } \\
\text { costs }[5,48] \\
\text { Good partial load } \\
\text { behaviour [47] }\end{array}$ & $\begin{array}{c}\text { Low quality } \\
\text { fuels can be } \\
\text { used for } \\
\text { externally driven } \\
\text { units [21] } \\
\text { Very few } \\
\text { moving parts } \\
\text { [21] } \\
\text { Very compact } \\
\text { sizes [21] } \\
\text { High } \\
\text { temperature } \\
\text { exhaust [45] } \\
\text { Low emissions } \\
\text { [45] }\end{array}$ \\
\hline Disadvantages & $\begin{array}{l}\text { Short maintenance } \\
\text { intervals }[21,45] \\
\text { Instability with bio- } \\
\text { and syngas/ limited } \\
\text { fuel flexibility } \\
{[21,48,49]} \\
\text { High noise [45] } \\
\text { High NOx- } \\
\text { emissions [5,21] } \\
\text { Using waste heat } \\
\text { difficult [45] }\end{array}$ & $\begin{array}{l}\text { Very high } \\
\text { investment } \\
\text { costs [5] } \\
\text { SOFCs have } \\
\text { long start-up } \\
\text { times [25] } \\
\text { Requires very } \\
\text { high quality, } \\
\text { energy intensive } \\
\text { fuels [21] } \\
\text { Low heat } \\
\text { recovery [5] }\end{array}$ & $\begin{array}{c}\text { Still high } \\
\text { investment costs } \\
{[21]} \\
\text { Low electric } \\
\text { efficiency }[5,32] \\
\text { Technology not } \\
\text { fully developed } \\
{[32,45]} \\
\text { Difficult to control } \\
\text { power output [32] }\end{array}$ & $\begin{array}{l}\text { Still high investment } \\
\text { costs [5] } \\
\text { Low maximal } \\
\text { electric efficiency [5] } \\
\text { Low heat recovery } \\
\text { for low-grade heat } \\
\text { applications [2] }\end{array}$ & $\begin{array}{c}\text { Still high } \\
\text { investment } \\
\text { costs [21] } \\
\text { Difficult Start-up } \\
{[5,21]} \\
\text { Delicate } \\
\text { mechanical } \\
\text { design [9] } \\
\text { Low electric } \\
\text { efficiency [18] }\end{array}$ \\
\hline $\begin{array}{c}\text { Commonness } \\
\text { in the reviewed } \\
\text { cases }\end{array}$ & $\begin{array}{c}\text { Most common } \\
19 / 41\end{array}$ & $\begin{array}{l}\text { Rare } \\
3 / 41\end{array}$ & $\begin{array}{l}\text { Rare } \\
2 / 41\end{array}$ & $\begin{array}{c}\text { Common } \\
12 / 41\end{array}$ & $\begin{array}{l}\text { Rare } \\
5 / 41\end{array}$ \\
\hline $\begin{array}{c}\text { Assessment of } \\
\text { potential }\end{array}$ & Remains stable & High [45] & Very High [2] & High [2] & $\begin{array}{c}\text { Very high } \\
{[21,50]}\end{array}$ \\
\hline
\end{tabular}




\subsection{Internal Combustion Engines}

Due to their advanced technological development, low initial investment costs and widespread distribution, internal combustion engines (ICEs) represent the most often applied and most researched prime mover. The nominal electric loads of ICEs can range from $3 \mathrm{~kW}$ to up to $100 \mathrm{MW}$, making them applicable for all sizes of small-scale CCHP system. In addition, the electric efficiency of ICEs can reach up to $45 \%$, but the thermal loses are comparatively high, so that the overall efficiency of ICE systems is generally equal or lower compared to other prime movers. In small-scale CCHP systems the amount of chemical energy converted into electric energy is found to be a bit lower, usually in the range of 30-35\% [51]. Especially in the context of syngas usage, ICEs are quickly damaged by low quality gases (i.e. sticky tar can clog injection nozzles) and hence their efficiency is lowered significantly $[48,49]$. Therefore, bigger CCHP systems using ICEs and direct gasification generally include more sophisticated cleaning systems [52]. All described systems of small-scale bio-fired CCHP systems with ICEs are summarized in Table 2-Table 5. Cases of small-scale bio-fired CCHP systems using ICEs in combination with solar extensions are summarized in Table 8. 
Table 2 Cases of small-scale biomass-fired CCHP systems with ICEs with 100-300 kW nominal power

\begin{tabular}{|c|c|c|c|c|c|c|}
\hline Ref. & $\begin{array}{l}\text { Max. } \\
\text { Outputs }\end{array}$ & Biomass & $\begin{array}{l}\text { Prime } \\
\text { mover }\end{array}$ & $\begin{array}{l}\text { Refrigerat } \\
\text { ion }\end{array}$ & $\begin{array}{l}\text { Software/ } \\
\text { Location }\end{array}$ & Key Findings \\
\hline [53] & $\begin{array}{l}\text { Electricity: } \\
250 \mathrm{~kW}- \\
1,974 \mathrm{~kW} \\
\text { Heating: } \\
2,057 \mathrm{~kW} \text { - } \\
5,217 \mathrm{~kW} \\
\text { Cooling: } \\
151 \mathrm{~kW}- \\
\text { no limit } \\
\text { specified }\end{array}$ & $\begin{array}{l}\text { Wood chips } \\
\text { and almond } \\
\text { shells } \\
\text { (gasified) }\end{array}$ & $\begin{array}{l}\text { ICE } \\
\text { (in the } \\
\text { referenc } \\
e \\
\text { system } \\
\text { with } 767 \\
k W \\
\text { nominal } \\
\text { power) }\end{array}$ & $A C$ & $\begin{array}{l}\text { EES Model } \\
\text { for a district } \\
\text { heating and } \\
\text { cooling } \\
\text { network }\end{array}$ & $\begin{array}{l}\text { Analysis of } 5 \text { different configurations: } \\
\text { - single vs. double AC } \\
\text { - Heat recovery after air preheating vs. } \\
\text { only engine exhaust gases } \\
\text { Double effect AC with both heat recover } \\
\text { inputs is the most efficient solution } \\
\text { Overall system energy efficiency: } 60 \% \\
\text { Electric efficiency: } 37 \%\end{array}$ \\
\hline [54] & $\begin{array}{l}\text { Electricity: } \\
250 \mathrm{~kW} \\
\text { Heating: } \\
320 \mathrm{~kW} \text { (in } \\
\text { trigeneration) } \\
\text { Cooling: } \\
92.2 \mathrm{~kW}\end{array}$ & $\begin{array}{l}\text { Gasification: } \\
\text { - willow chips } \\
\text { - Miscanthus } \\
\text { - rice husk }\end{array}$ & ICE & $\begin{array}{l}\text { Ammonia } \\
\text { absorption }\end{array}$ & $\begin{array}{l}\text { Model in } \\
\text { Eclipse }\end{array}$ & $\begin{array}{l}\text { Energetic Efficiencies: } \\
\text { CHP 61.4-66.5\% } \\
\text { Trigeneration: } 53.5 \%-58.6 \% \\
\text { Cogeneration produces least amount of } \\
\mathrm{CO}_{2} \text {-emissions } \\
\text { Difference in biomass inputs affects } \\
\text { technical performance marginally, but has } \\
\text { great impact on economics due to biomass } \\
\text { cost } \\
\text { Use of Miscanthus leads to highest } \\
\text { efficiencies } \\
\text { All three biomasses economically feasible } \\
\text { in the proposed trigeneration system }\end{array}$ \\
\hline [55] & $\begin{array}{l}\text { Electricity: } \\
240 \mathrm{~kW} \\
\text { Heating: } \\
225 \mathrm{~kW} \\
\text { Cooling: } \\
13 \mathrm{~kW}\end{array}$ & Jatropha Oil & ICE & $\begin{array}{l}\text { Ammonia } \\
A C\end{array}$ & $\begin{array}{l}\text { Model in } \\
\text { ECLIPSE for } \\
\text { households } \\
\text { in GB }\end{array}$ & $\begin{array}{l}\text { Efficiencies of Jatropha oil fueled system } \\
\text { lower than diesel fueled } \\
\text { If emissions of the Jatropha oil were not } \\
\text { considered as net zero the } \mathrm{CO}_{2} \text { emissions } \\
\text { were found to be higher compared to } \\
\text { diesel } \mathrm{CO}_{2} \text { emissions (kg/kWh) less than } \\
\text { half compared to power-only mode }\end{array}$ \\
\hline [56] & $\begin{array}{l}\text { Electricity: } \\
120 \mathrm{~kW} \\
\text { Heating: } \\
\text { up to } 200 \mathrm{~kW} \\
\text { Cooling: } \\
25.6 \mathrm{~kW}\end{array}$ & $\begin{array}{l}\text { Biogas through } \\
\text { anaerobic } \\
\text { digestion of } \\
\text { cow manure } \\
\text { and brewers } \\
\text { waste }\end{array}$ & $\begin{array}{l}\text { Lister- } \\
\text { Petter } \\
\text { diesel } \\
\text { engine } \\
\text { assisted } \\
\text { by dual- } \\
\text { fuel } \\
\text { boiler }\end{array}$ & $\begin{array}{l}\text { Gas-fired } \\
\text { ammonia- } \\
\text { water AC }\end{array}$ & $\begin{array}{l}\text { Eclipse } \\
\text { software for } \\
\text { a micro- } \\
\text { brewery in } \\
\text { England }\end{array}$ & $\begin{array}{l}\text { CHP system feasible under national funding } \\
\text { scheme } \\
\text { Trigeneration system calculated to have a } \\
\text { payback period of } 5.4 \text { years and the highest } \\
\text { additional income over } 20 \text { years }\end{array}$ \\
\hline [57] & $\begin{array}{l}\text { Electricity: } \\
\text { designed for } \\
\text { 100kW } \\
\text { Heating: } \\
\sim 131 \mathrm{~kW} \\
\text { Cooling: } \\
\sim 76 \mathrm{~kW}\end{array}$ & $\begin{array}{l}\text { Wood chips } \\
\text { (gasified) } \\
+ \text { natural gas }\end{array}$ & ICE & $A C$ & $\begin{array}{l}\text { Model for a } \\
\text { Chinese } \\
\text { region } \\
\text { supported } \\
\text { by EES }\end{array}$ & $\begin{array}{l}\text { Combined use of natural gas and wood } \\
\text { chips for a CCHP system } \\
\text { Cofiring syngas and natural gas has higher } \\
\text { efficiency than just combusting syngas } \\
\text { Energetic efficiency } 70-79.5 \% \\
\text { Exergetic efficiency: } 22-35.6 \% \\
\text { Costs heavily affected by biomass and natural } \\
\text { gas price }\end{array}$ \\
\hline
\end{tabular}


Table 3 Cases of small-scale biomass-fired CCHP systems with ICEs with 300-1000 kW nominal power

\begin{tabular}{|c|c|c|c|c|c|c|}
\hline Ref. & $\begin{array}{l}\text { Max. } \\
\text { Outputs }\end{array}$ & Biomass & $\begin{array}{l}\text { Prime } \\
\text { mover }\end{array}$ & $\begin{array}{l}\text { Refrigerat } \\
\text { ion }\end{array}$ & $\begin{array}{l}\text { Software/ } \\
\text { Location }\end{array}$ & Key Findings \\
\hline $\begin{array}{l}{[58]} \\
{[59]}\end{array}$ & $\begin{array}{l}\text { Electricity: } \\
446 \mathrm{~kW} \\
\text { Chilled } \\
\text { Water: } \\
1,804 \mathrm{~kW} \\
\text { Heating: } \\
595 \mathrm{~kW} \\
\text { Hot Water: } \\
335 \mathrm{~kW}\end{array}$ & $\begin{array}{l}\text { Gasification: } \\
\text { Straw } \\
(0.297 \mathrm{~kg} / \mathrm{s})\end{array}$ & ICE & $\begin{array}{l}\text { Two-Stage } \\
\text { LiBr- } \mathrm{H}_{2} \mathrm{O} \\
\text { Absorption } \\
\text { chiller } \\
\text { (AC) }\end{array}$ & $\begin{array}{l}\text { DEST } \\
\text { software }\end{array}$ & $\begin{array}{l}\text { Efficiencies: } \\
\text { Summer/ Winter/ Transition: } \\
\text { Energy: } 50 \% / 37.8 \% / 37 \% \\
\text { Exergy: } 6.23 \% / 12.51 \% / 13.79 \% \\
\text { Biomass costs greatly influence unit costs of } \\
\text { outputs } \\
\text { Greatest exergy destruction in gasification } \\
\text { system ( 70\%) }\end{array}$ \\
\hline $\begin{array}{l}{[60]} \\
{[61]}\end{array}$ & $\begin{array}{l}\text { Electricity: } \\
\text { 1,000 kW } \\
\text { Heating: } \\
\text { 2,000 kW } \\
\text { Cooling: } \\
2,800 \mathrm{~kW} \\
\text { (rated } \\
\text { refrigerating } \\
\text { capacity) }\end{array}$ & $\begin{array}{l}\text { Anaerobic } \\
\text { digestion: } \\
\text { - straw } \\
\text { - guano } \\
\text { - kitchen } \\
\text { waste } \\
\text { - sludge } \\
\text { Gasification } \\
\text { - Wood } \\
\text { - Coconut } \\
\text { - straw }\end{array}$ & $\begin{array}{l}\text { ICE } \\
\text { (gas) }\end{array}$ & $A C$ & Model & $\begin{array}{l}\text { More } \mathrm{CH}_{4} \text { in anaerobic digested gases, more } \\
\mathrm{H}_{2} \text { in gasified gases } \\
\text { Anaerobic digested gases generates more } \\
\text { electricity and less heat } \\
\text { For both gasification and anaerobic digestion } \\
\text { optimal values were calculated for output } \\
\text { work of engine, compression ratio, PESR and } \\
\text { exergy efficiency with different temperature } \\
\text { ratio and exhaust gas energy level }\end{array}$ \\
\hline [23] & $\begin{array}{l}\text { Electricity: } \\
346 \mathrm{~kW} \\
\text { Heating: } \\
1,063 \mathrm{~kW} \\
\text { Cooling: } \\
\text { 1,010 kW }\end{array}$ & $\begin{array}{l}\text { Wood chips } \\
\text { (gasified) }\end{array}$ & $\begin{array}{l}\text { Not } \\
\text { specified }\end{array}$ & $A C$ & $\begin{array}{l}\text { MATLAB } \\
\text { model for } \\
\text { a travel } \\
\text { hotel in } \\
\text { Harbin, } \\
\text { China }\end{array}$ & $\begin{array}{l}\text { Compared to separated production: } \\
\text { - } 90.4 \% \mathrm{CO}_{2} \text { saved } \\
\text { - } 17.7 \% \text { higher primary energy consumption } \\
\text { due to lower energetic efficiency } \\
\text { - Annual total cost saving: } 45.4 \%\end{array}$ \\
\hline
\end{tabular}


Table 4 Cases of small-scale biomass-fired CCHP systems with ICEs with less than $40 \mathrm{~kW}$ nominal power

\begin{tabular}{|c|c|c|c|c|c|c|}
\hline Ref. & $\begin{array}{c}\text { Max. } \\
\text { Outputs }\end{array}$ & Biomass & $\begin{array}{l}\text { Prime } \\
\text { mover }\end{array}$ & Refrigeration & $\begin{array}{l}\text { Software/ } \\
\text { Location }\end{array}$ & Key Findings \\
\hline [62] & $\begin{array}{l}\text { Electricity: } \\
9.9 \mathrm{~kW} \\
\text { Heating: } \\
4.5 \mathrm{~kW} \\
\text { Ice: } \\
40 \mathrm{~kg} / \mathrm{h}=4 \mathrm{~kW} \\
\text { Clean water }\end{array}$ & $\begin{array}{l}\text { Jatropha and } \\
\text { Pongamia oils } \\
(3 L / h)\end{array}$ & $\begin{array}{l}\text { ICE } \\
\text { (Diesel - } \\
\text { Lister } \\
\text { Petter CI } \\
\text { Engine) }\end{array}$ & $\begin{array}{l}\text { Adsorption } \\
\text { refrigerator }\end{array}$ & Experiment & $\begin{array}{l}\text { Thermal efficiency: } 63 \% \\
\text { Payback: } 1.7 \text { - } 3.2 \text { years } \\
\text { Low GHG emissions but also low } \\
\text { COP with adsorption refrigerator } \\
\text { Clean water produced through } \\
\text { multiple-effect distillation }\end{array}$ \\
\hline [63] & $\begin{array}{l}\text { Electricity: } \\
6.5 \mathrm{~kW} \\
\text { Hot Water: } \\
\sim 12.3 \mathrm{~kW} \\
\text { Cooling: } \\
15 \mathrm{~W}\end{array}$ & \begin{tabular}{|l|} 
Croton \\
megalocarpus \\
oil
\end{tabular} & $\begin{array}{l}\text { Dual-use } \\
\text { ICE } \\
\text { (compre } \\
\text { ssion } \\
\text { ignition) }\end{array}$ & $\begin{array}{l}A C \\
+P C M \\
\text { storage }\end{array}$ & Experiment & $\begin{array}{l}\text { Overall efficiency: } 76 \% \text { with bio oil } \\
\text { (compared to } 88 \% \text { with diesel) } \\
\text { Electric efficiency: } 25-30 \% \\
\text { Higher specific fuel consumption } \\
\text { with bio oil compared to diesel due } \\
\text { to maladjusted injection settings } \\
\text { More heat recovery with biooil due } \\
\text { to longer injection duration } \\
\text { Preheating could lead to higher } \\
\text { efficiency and less particulate } \\
\text { emission }\end{array}$ \\
\hline [64] & $\begin{array}{l}\text { Electricity: } \\
15 \mathrm{~kW} \\
\\
\text { Heating: } \\
\sim 17 \mathrm{~kW} \\
\text { Cooling: } \\
\sim 4 \mathrm{~kW} \text { (in } \\
\text { trigeneration } \\
\text { mode) }\end{array}$ & \begin{tabular}{|l|} 
Ligno- \\
celulosic/ \\
eucaliptus \\
wood \\
(20 to $30 \mathrm{~kg} / \mathrm{h})$ \\
gasified in a \\
downdraft \\
gasifier
\end{tabular} & ICE & $L i B r-A C$ & $\begin{array}{l}\text { Analysis } \\
\text { based on } \\
\text { experiments } \\
\text { in Zaragoza, } \\
\text { Spain }\end{array}$ & $\begin{array}{l}\text { Highest energy efficiency achieved: } \\
51.42 \% \\
\text { Feasible as alternative for rural } \\
\text { areas with shortage of petroleum } \\
\text { and abundance of biomass } \\
\text { Electric efficiency of } 21.42 \%\end{array}$ \\
\hline [65] & $\begin{array}{l}\text { Reference } \\
\text { system (summer } \\
\text { mode): } \\
\\
\text { Electricity: } \\
1.5 \mathrm{~kW} \\
\\
\text { Heating: } \\
19.6 \mathrm{~kW} \\
\text { Cooling: } \\
9.1 \mathrm{~kW}\end{array}$ & $\begin{array}{l}\text { B-100 } \\
\text { biodiesel }\end{array}$ & ICE & $\begin{array}{l}\text { Vapour } \\
\text { compression } \\
\text { chiller }\end{array}$ & $\begin{array}{l}\text { Thermo- } \\
\text { dynamic } \\
\text { model based } \\
\text { on data from } \\
\text { experiments } \\
\text { and literature }\end{array}$ & $\begin{array}{l}\text { Comparison between conventional } \\
\text { diesel and biodiesel: } \\
\text { - Primary energy consumption } \\
\text { decreased by }-50 \% \\
\mathrm{CO}_{2} \text { emissions by } \sim 95 \% \\
\begin{array}{l}\text { Case study for a commercial building } \\
\text { complex in Hong Kong suggest a } \\
\text { switch to heat-driven chillers }\end{array}\end{array}$ \\
\hline
\end{tabular}


Table 5 Cases of small-scale biomass-fired CCHP systems with ICEs with 40-100 kW nominal power

\begin{tabular}{|c|c|c|c|c|c|c|}
\hline Ref. & $\begin{array}{c}\text { Max. } \\
\text { Outputs }\end{array}$ & Biomass & $\begin{array}{l}\text { Prime } \\
\text { mover }\end{array}$ & Refrigeration & $\begin{array}{l}\text { Software/ } \\
\text { Location }\end{array}$ & Key Findings \\
\hline [66] & $\begin{array}{l}\text { Electricity: } \\
\sim 40 \mathrm{~kW} \\
\text { Heating: } \\
\sim 60 \mathrm{~kW} \\
\text { Cooling: } \\
\text { not specified }\end{array}$ & \begin{tabular}{|l|} 
Biomass- \\
derived gases \\
(gasification \\
vs. pyrolysis \\
vs. anaerobic \\
digestion) \\
mixed with \\
LPG
\end{tabular} & $\begin{array}{l}\text { ICE } \\
\text { (gas) }\end{array}$ & $\begin{array}{l}\text { Mixed effect } \\
\text { AC }\end{array}$ & $\begin{array}{l}\text { Mathematical } \\
\text { Model }\end{array}$ & $\begin{array}{l}\text { Addition of LPG to all three types of } \\
\text { gases increases exergy and } \\
\text { energy efficiency and decreases } \\
\mathrm{CO}_{2} \text { emissions for gases from } \\
\text { gasification and pyrolysis, but from } \\
\text { anaerobic digestion } \\
\text { Lower heating value and air fuel } \\
\text { ratio of the biomass-derived gas } \\
\text { affects the impact of } L P G \text { the most }\end{array}$ \\
\hline $\begin{array}{l}\text { [67] } \\
{[68]}\end{array}$ & $\begin{array}{l}\text { Electricity: } \\
50 \mathrm{~kW} \\
\text { Heating: } \\
120 \mathrm{~kW} \\
\text { Cooling: } \\
20 \mathrm{~kW} \text { (in } \\
\text { optimized case) }\end{array}$ & $\begin{array}{l}\text { Wood chips } \\
\text { (gasified by } \\
\text { fixed bed } \\
\text { gasifier) }\end{array}$ & ICE & $\begin{array}{l}A C \\
(C O P: 0.8)\end{array}$ & $\begin{array}{l}\text { Matlab model } \\
\text { for Italian } \\
\text { building } \\
\text { cluster }\end{array}$ & $\begin{array}{l}\text { Electrical efficiency: } 20.6 \% \\
\text { Thermal efficiency: } 40.5 \% \\
\text { Upgrade of a CHP system } \\
\text { Thermal energy storage (of not } \\
\text { specified type) considered, but has } \\
\text { a negligent effect on economic } \\
\text { performance } \\
\text { Size of AC is the most important } \\
\text { factor for feasible investment }\end{array}$ \\
\hline [69] & $\begin{array}{l}\text { Electricity: } \\
56 \mathrm{~kW} \text { (shaft } \\
\text { power) } \\
\text { Heating: } \\
\sim 110 \mathrm{~kW} \\
\text { Cooling: } \\
\sim 60 \mathrm{~kW}\end{array}$ & $\begin{array}{l}\text { LPG mixed } \\
\text { with biomass- } \\
\text { derived gases: } \\
\text { - Gasification } \\
\text { - Pyrolysis } \\
\text { - Anaerobic } \\
\text { digestion }\end{array}$ & ICE & $\begin{array}{l}\text { Mixed effect } \\
\text { absorption } \\
\text { chiller (COP: } \\
\text { up to 0.94) }\end{array}$ & $\begin{array}{l}\text { Mathematical } \\
\text { model (no } \\
\text { software } \\
\text { mentioned) }\end{array}$ & $\begin{array}{l}\text { Electrical efficiency: } 30 \% \\
\text { Simulating the effects of adding LPG } \\
\text { to biomass-derived gases: } \\
\text { - better energetic and exergetic } \\
\text { performance for gasification and } \\
\text { pyrolysis derived gases } \\
\text { - worse energetic and exergetic } \\
\text { performance for gases from } \\
\text { anaerobic digestion }\end{array}$ \\
\hline
\end{tabular}


Table 6 Cases of small-scale biomass-fired and solar-assisted CCHP systems with ICEs

\begin{tabular}{|c|c|c|c|c|c|c|}
\hline Ref. & $\begin{array}{c}\text { Max. } \\
\text { Outputs }\end{array}$ & Biomass & $\begin{array}{l}\text { Prime } \\
\text { mover }\end{array}$ & $\begin{array}{l}\text { Refrigerat } \\
\text { ion }\end{array}$ & $\begin{array}{l}\text { Software/ } \\
\text { Location }\end{array}$ & Key Findings \\
\hline [71] & $\begin{array}{l}\text { Electricity: } \\
987 \mathrm{~kW} \\
\text { Hot water: } \\
1,988 \mathrm{~kW} \\
\text { Cooled } \\
\text { Water: } \\
843 \mathrm{~kW} \\
\text { Dry Air: } \\
482 \mathrm{~kW}\end{array}$ & $\begin{array}{l}\text { Rice husk } \\
(1,400 \mathrm{~kg} / \mathrm{h} \\
\text { - gasified) }\end{array}$ & $\begin{array}{l}\text { Gas ICE } \\
+ \\
\text { solar } \\
\text { collectors } \\
\text { connected } \\
\text { to } \\
\text { gasification }\end{array}$ & $\begin{array}{l}\mathrm{LiBr}-\mathrm{H}_{2} \mathrm{O} \\
\mathrm{AC} \\
+ \text { liquid } \\
\text { desiccant } \\
\text { cooler }\end{array}$ & $\begin{array}{l}\text { Aspen } \\
\text { plus }\end{array}$ & $\begin{array}{l}\text { Most exergy destroyed in gasifier and engine } \\
\text { Exergy efficiency: } 19.21 \% \\
\text { Energy efficiency: } 77.4 \% \text { (when only } \\
\text { considering positive influence of solar energy) } \\
\text { Electric efficiency: } 15 \% \\
\text { Air equivalence ratio, steam/biomass ratio and } \\
\text { air preheating have significant effects on } \\
\text { gasification } \\
\text { Solar thermal energy can decrease biomass } \\
\text { consumption by } 29 \% \\
\text { Annual cost savings of up to } 25.9 \% \text { compared } \\
\text { to separate systems }\end{array}$ \\
\hline $\begin{array}{l}{[72]} \\
{[73]}\end{array}$ & $\begin{array}{l}\text { Electricity: } \\
\text { 1,000 kW } \\
\text { Heating: } \\
580.2 \mathrm{~kW} \\
\text { Cooling: } \\
67.9 \mathrm{~kW}\end{array}$ & $\begin{array}{l}\text { Biogas } \\
\text { from cattle } \\
\text { and pig } \\
\text { manure }\end{array}$ & $\begin{array}{l}\text { ICE } \\
(750 \mathrm{kWe}) \\
+ \\
P V \text { cells } \\
(255 \mathrm{kWe})\end{array}$ & $\begin{array}{l}\text { Adsorption } \\
\text { Chiller }\end{array}$ & $\begin{array}{l}\text { Model } \\
\text { (example } \\
\text { given for } \\
\text { an } \\
\text { agricultur } \\
\text { al } \\
\text { process } \\
\text { facility) }\end{array}$ & $\begin{array}{l}\text { Thermoeconomic analysis (TEA) and } \\
\text { thermos-ecological analysis (TEC) } \\
\text { Exergy efficiency of adsorption chiller } \\
\text { considerably low } \\
\text { Electric efficiency of ICE: } 47.9 \% \\
\text { ICE provides } 64.7 \% \text { and PV panels } 8.9 \% \text { of } \\
\text { consumer's electricity demand, rest is } \\
\text { provided by electric grid }\end{array}$ \\
\hline [74] & $\begin{array}{l}\text { Electricity: } \\
100 \mathrm{~kW} \\
\text { Hot water: } \\
102.7 \mathrm{~kW} \\
\\
\text { Cooled } \\
\text { water: } \\
197.2 \mathrm{~kW}\end{array}$ & $\begin{array}{l}\text { Wood } \\
\text { chips } \\
\text { (gasified) }\end{array}$ & $\begin{array}{l}\text { ICE (gas) } \\
+ \\
\text { solar- } \\
\text { evacuated } \\
\text { thermal } \\
\text { collectors }\end{array}$ & $\begin{array}{l}\text { Mixed } \\
\text { effect LiBr- } \\
\text { AC (using } \\
\text { engine air } \\
\text { exhaust } \\
\text { and solar } \\
\text { hot water) } \\
\text { ( COP 1) }\end{array}$ & $\begin{array}{l}\text { EES } \\
\text { Model }\end{array}$ & $\begin{array}{l}\text { Solar collectors to heat up waste stream after } \\
\text { ICE for absorption chilling } \\
\text { Using a higher biomass to solar energy ratio } \\
\text { leads to higher efficiency but also to more } \\
\text { carbon emissions } \\
\text { Exergetic efficiency mostly influenced by } \\
\text { biomass subsystem } \\
\text { Overall system energy efficiency: } 57.9 \% \\
\text { Solar subsystem energy efficiency: } 47 \% \\
\text { Bio subsystem energy efficiency: } 61 \%\end{array}$ \\
\hline [75] & $\begin{array}{l}\text { Electricity: } \\
95 \mathrm{~kW} \\
\text { Heating: } \\
\text { unspecified } \\
\text { Cooling: } \\
325 \mathrm{~kW}\end{array}$ & $\begin{array}{l}\text { Vegetable } \\
\text { oil } \\
\text { (rapeseed } \\
\text { oil) }\end{array}$ & $\begin{array}{l}\text { ICE + } \\
\text { parabolic } \\
\text { through } \\
\text { collectors }\end{array}$ & $\begin{array}{l}\mathrm{LiBr}-\mathrm{H}_{2} \mathrm{O} \\
\text { double- } \\
\text { stage } A C \\
(\sim \mathrm{COP} \\
1.4)\end{array}$ & $\begin{array}{l}\text { Model in } \\
\text { TRNSYS }\end{array}$ & $\begin{array}{l}\text { Solar collectors to heat up waste stream after } \\
\text { ICE for absorption chilling } \\
\text { PESR: } 93 \% \\
\text { Discounted payback period of around } 9 \text { years, } \\
\text { highly dependent on pure plant oil price }\end{array}$ \\
\hline
\end{tabular}

\subsection{Fuel Cells}

With no moving parts and no combustion, fuel cells (FCs) are a completely different type of prime mover from their mechanical counterparts, excelling in environmental impact and load flexibility [45]. However, the environmental benefit is rapidly equalized when considering the energy consumption and GHG emissions for producing hydrogen or methane as fuel. FCs generally have a high electric efficiency and thus a lower heat recovery and bio-fueled CCHP systems using FCs which can serve loads from less than $1 \mathrm{~kW}$ to more than $1,000 \mathrm{~kW}$ have been demonstrated [5,21,76,77]. A huge disadvantage for FCs is the need of very high fuel purity levels. Research is therefore being conducted in techniques to remove 
sulphur, halogens, alkali and siloxanes from biogas, while for gasification systems, an efficient technology to remove tar is still the main obstacle for commercialization with investigations still ongoing [78-81]. Table 7 shows a summary of the investigated small-scale biomass-fired and solarassisted CCHP systems. There is also a case of system with an Organic Rankine Cycle (ORC) enhanced by a FC (see Table 11).

Table 7 Cases of small-scale biomass-fired and solar-assisted CCHP systems with fuel cells

\begin{tabular}{|c|c|c|c|c|c|c|}
\hline Ref. & $\begin{array}{c}\text { Max. } \\
\text { Outputs }\end{array}$ & Biomass & $\begin{array}{l}\text { Prime } \\
\text { mover }\end{array}$ & Refrigeration & $\begin{array}{l}\text { Software/ } \\
\text { Location }\end{array}$ & Key Findings \\
\hline [82] & $\begin{array}{l}\text { Electricity: } \\
\text {-220 kW } \\
\text { Heating: } \\
69.9 \mathrm{~kW} \\
\\
\text { Cooling: } \\
22.1 \mathrm{~kW}\end{array}$ & $\begin{array}{l}\text { Not specified } \\
\text { (gasification) }\end{array}$ & $\begin{array}{l}\text { SOFC + } \\
\text { ORC } \\
\text { turbine } \\
\text { (with } \\
\text { toluene) }\end{array}$ & $\begin{array}{l}\text { LiBr-AC } \\
(\sim C O P \text { C.7- } \\
0.8)\end{array}$ & $\begin{array}{l}\text { EES } \\
\text { software }\end{array}$ & $\begin{array}{l}\text { Energy : } 78.8 \% \\
\text { Exergy efficiency: } 50.6 \% \\
\text { Comparing various gaseous fuels from } \\
\text { different gasifier systems: } \\
\text { 1.Bubbling fluidized bed } \\
\text { 2. Circulating fluidized bed } \\
\text { 3.Dual fluidized bed } \\
\text { Syngas from bubbling fluidized bed } \\
\text { gasifier shows highest thermodynamic } \\
\text { performance with a net system energy } \\
\text { efficiency of } 60 \%\end{array}$ \\
\hline $\begin{array}{l}{[83]} \\
{[84]} \\
{[85]}\end{array}$ & $\begin{array}{l}\text { Electricity: } \\
\sim 329 \mathrm{~kW} \\
\text { SOFC } \\
+\sim 89 \mathrm{~kW} \\
\text { ORC } \\
\text { Heating: } \\
\sim 646 \mathrm{~kW} \\
\text { Cooling: } \\
\sim 51 \mathrm{~kW}\end{array}$ & $\begin{array}{l}\text { Syngas from } \\
\text { gasification } \\
\text { (no more } \\
\text { specification) }\end{array}$ & $\begin{array}{l}\text { SOFC } \\
\text { and ORC } \\
\text { with two } \\
\text { turbines } \\
+ \\
\text { parabolic } \\
\text { through } \\
\text { solar } \\
\text { collectors } \\
\text { super- } \\
\text { heating } \\
\text { ORC } \\
\text { circuit }\end{array}$ & $L i B r-A C$ & $\begin{array}{l}\text { EES } \\
\text { software }\end{array}$ & $\begin{array}{l}\text { Highest system energy efficiency: } 85.1 \% \\
\text { Highest system exergy efficiency: } 32.62 \% \\
\text { Highest exergetic losses in SOFC, ORC } \\
\text { evaporator and solar panels } \\
\text { Solar assistance increases efficiency by } \\
\text { up to } 16 \% \text { and adds } 89 \mathrm{~kW} \text { electricity } \\
\text { generation } \\
\text { SOFC input parameters (fuel utilization } \\
\text { ratio, recirculation ratio, stack temperature) } \\
\text { influence system significantly } \\
\text { Exergetic efficiency up to } 25 \% \text { higher } \\
\text { compared to power-only cycle }\end{array}$ \\
\hline [86] & $\begin{array}{l}\text { Electricity: } \\
630 \mathrm{~kW} \\
\text { Heating: } \\
1185 \mathrm{~kW} \\
\text { Cooling: } \\
320 \mathrm{~kW}\end{array}$ & $\begin{array}{l}\text { Downdraft } \\
\text { gasification } \\
\text { from } \\
\text { lignocellulosic } \\
\text { biomass } \\
\text { (pine wood } \\
\text { residues) }\end{array}$ & $\begin{array}{l}\text { SOFC } \\
+ \text { Solar } \\
\text { thermal } \\
\text { collectors } \\
\text { for } \\
\text { heating }\end{array}$ & $\begin{array}{l}\text { Silica-Gel } \\
\text { Adsorption } \\
\text { chiller (and } \\
\text { electric } \\
\text { chiller) }\end{array}$ & $\begin{array}{l}\text { ASPEN } \\
\text { model with } \\
\text { TRNSYS } \\
\text { environment }\end{array}$ & $\begin{array}{l}\text { Total energy efficiency: } 56 \% \\
\text { Electric efficiency: } 23 \% \\
\text { Three different system configurations: } \\
\text { SOFC + adsorption chiller } \\
\text { SOF + electric chiller } \\
\text { SOFC + electric chiller + solar collectors } \\
\text { Hot water storage considered in all cases } \\
\text { Adsorption chiller based on } 10 \mathrm{~kW} \\
\text { commercial unit } \\
\text { Low efficiency due to cold gas efficiency in } \\
\text { gasifier } \\
\text { PSER: } 50 \% \\
\mathrm{CO}_{2} \text {-emissions savings: } 5000 \text { t/y }\end{array}$ \\
\hline
\end{tabular}

\subsection{Stirling Engines}

Compared to the previously mentioned ICEs, Stirling engines (SEs) have the potential to operate more quietly, with better partial load performance and low maintenance costs due to less moving parts $[12,48,87]$. They can satisfy electric loads from a few $\mathrm{kW}$ up to $150 \mathrm{~kW}$, while also being able to provide high temperature heat exhausts with high thermal efficiency $[2,21,88]$. Especially in the context of 
biomass usage, SEs show great fuel flexibility being able to be driven by direct combustion of biomass or by combustion of syn- or biogas, because the heat can be applied externally [89]. Uncertainties in operating SEs and high investment costs hinder further use of SE technology [89]. A summary of the investigated small-scale bio CCHP systems with Stirling engines is given in Table 8.

There are few cases using SEs as prime movers in the context of small-scale CHP and CCHP systems, which however report promising results for reducing GHG emissions due to high energetic efficiencies [89-97]. However only few cases of such systems fueled explicitly with biomass could be found within the literature of scientific journals, which are summarized in Table 8.

Table 8 Cases of small-scale biomass-fired CCHP systems with Stirling engines

\begin{tabular}{|c|c|c|c|c|c|c|}
\hline Ref. & $\begin{array}{c}\text { Max. } \\
\text { Outputs }\end{array}$ & Biomass & $\begin{array}{l}\text { Prime } \\
\text { mover }\end{array}$ & Refrigeration & $\begin{array}{l}\text { Software/ } \\
\text { Location }\end{array}$ & Key Findings \\
\hline [98] & $\begin{array}{l}\text { SE: } \\
\text { Electricity: } \\
3.2 \mathrm{~kW} \\
\text { Heating: } \\
10.9 \mathrm{~kW} \\
\text { Cooling: } \\
4.4 \mathrm{~kW} \\
\\
\text { Compared } \\
\text { with an ICE: } \\
\text { Electricity: } \\
5.2 \mathrm{~kW} \\
\text { Heating: } \\
7.6 \mathrm{~kW} \\
\text { Cooling: } \\
2.2 \mathrm{~kW}\end{array}$ & $\begin{array}{l}\text { Option 1: } \\
\text { Wood pellets } \\
\text { SE } \\
\text { Option 2: } \\
\text { willow chips } \\
\text { SE } \\
\text { Option 3: } \\
\text { biodiesel ICE }\end{array}$ & $\begin{array}{l}\text { SE vS. } \\
I C E\end{array}$ & $L i B r-A C$ & $\begin{array}{l}\text { Model in } \\
\text { Eclipse }\end{array}$ & $\begin{array}{l}\text { SE: Overall efficiency } 67 \% \text { (electric } \\
\text { efficiency 20\%) } \\
\text { ICE: Overall efficiency } 60.1 \% \text { (but } \\
\text { higher electric efficiency with 30\%) } \\
\text { Potential of saving } 6.1 \text { to } 8.9 \text { tonnes } \\
\text { of } \mathrm{CO}_{2} \text { per year } \\
\text { Batteries with a storage capacity of } 13 \\
\text { kWh were also modelled into the } \\
\text { system } \\
\text { SE option more feasible when } \\
\text { electricity/heat demand ration is low, } \\
\text { otherwise ICE option more feasible }\end{array}$ \\
\hline [88] & $\begin{array}{l}\text { Electricity: } \\
\text { 0-15 kW } \\
\text { Heating: } \\
\text { not specified } \\
\text { Cooling: } \\
\text { not specified }\end{array}$ & Wood chips & SE & $A C$ & $\begin{array}{l}\text { EnergyPlus } \\
\text { software } \\
\text { using data } \\
\text { for a small } \\
\text { office in } \\
\text { Atlanta, } \\
\text { GA, USA }\end{array}$ & $\begin{array}{l}\text { Reference case with } 7 \mathrm{~kW} \mathrm{SE} \\
\text { Analysis of primary energy } \\
\text { consumption and operational costs } \\
\text { compared to the reference system } \\
\text { Identifying SE size, heat exchanger, } \\
\text { AC, and fuel costs as most important } \\
\text { variables for economy }\end{array}$ \\
\hline
\end{tabular}

\subsection{Organic Rankine Cycles}

Compared to ICE and conventional turbines, Organic Rankine Cycles (ORCs) perform better when run under partial load, although there can be significant losses [48]. ORCs are a potential key technology for achieving successful commercialization of small-scale CCHP systems [87]. ORCs have been proven to successfully satisfy loads from $100 \mathrm{~kW}$ up to $2 \mathrm{MW}$, while even smaller systems are currently researched in a pre-commercial status [47,99]. Larger systems can reach electric efficiencies of up to $35 \%$ and overall energetic efficiencies of around $85 \%$, but systems smaller than $100 \mathrm{~kW}$ reach only lower electric efficiencies of about 15\% [100]. A key decision for designing an ORC system is the choice of the organic working fluid, where several characteristics have to be considered: heat transfer properties, environmental impact, safety issues, chemical stability, pressure requirements, costs and availability, molecular weight, freezing point, curve of saturation, thermodynamic performance, corrosiveness $[47,99,101]$. A detailed overview of working fluids for low-temperature organic Rankine cycles can be found in Saleh et al. [102]. As mentioned before, several studies tested the combination of ORC with FCs (s. chapter4.2), but there are also numerous studies on small-scale CCHP systems relying solely on ORCs. In Table 9-Table 11, the cases of small-scale biomass-fired and solar-assisted CCHP systems using ORCs are summarized. 
Table 9 Cases of small-scale biomass-fired and solar-assisted CCHP systems with ORCs with less than $50 \mathrm{~kW}$ nominal power

\begin{tabular}{|c|c|c|c|c|c|c|}
\hline Ref. & $\begin{array}{c}\text { Max. } \\
\text { Outputs }\end{array}$ & Biomass & $\begin{array}{l}\text { Prime } \\
\text { mover }\end{array}$ & Refrigeration & $\begin{array}{l}\text { Software/ } \\
\text { Location }\end{array}$ & Key Findings \\
\hline [103] & $\begin{array}{l}\text { Electricity: } \\
0.5 \mathrm{~kW} \\
\text { Heating: } \\
9.6 \mathrm{~kW} \\
\text { Cooling: } \\
6.5 \mathrm{~kW}\end{array}$ & $\begin{array}{l}\text { Wood } \\
\text { pellets } \\
\text { (direct } \\
\text { combustion) }\end{array}$ & ORC turbine & $\begin{array}{l}\text { Liquid } \\
\text { desiccant } \\
\text { cooler } \\
\text { (Potassium } \\
\text { formate - } \\
\text { HCOOK) }\end{array}$ & Experiment & $\begin{array}{l}\text { Overall energetic efficiency of } \\
\text { CHP: } 83 \% \\
\text { Overall energetic efficiency of } \\
\text { trigeneration: } 84.4 \% \\
\text { Thermal/electric COP of cooler: } \\
0.86 / 7.7 \\
\text { Working fluid: HFE7100 (non- } \\
\text { flammable, non-toxic, low GHG } \\
\text { potential) }\end{array}$ \\
\hline [104] & $\begin{array}{l}\text { Electricity: } \\
1,42 \mathrm{~kW} \\
\text { Heating: } \\
53,5 \mathrm{~kW} \\
\text { Cooling: } \\
5 \mathrm{~kW}\end{array}$ & $\begin{array}{l}\text { SRC willow } \\
\text { pellet (direct } \\
\text { combustion) }\end{array}$ & $\begin{array}{l}\text { ORC } \\
\text { expander } \\
\text { (R245fa) } \\
+ \\
\text { parabolic- } \\
\text { through } \\
\text { collectors } \\
\text { (super- } \\
\text { heating after } \\
\text { reactor) }\end{array}$ & $\begin{array}{l}\text { Vapour } \\
\text { compression } \\
\text { chiller }\end{array}$ & $\begin{array}{l}\text { EES Model } \\
\text { for a } \\
\text { household }\end{array}$ & $\begin{array}{l}\text { Payback: } 7 \text { years (average case) } \\
\text { Estimated exergy efficiency of } \\
\text { about } 7 \% \\
\text { Energetic and economic analysis } \\
\text { distinguishing between summer } \\
\text { and winter mode }\end{array}$ \\
\hline [105] & $\begin{array}{l}\text { Electricity: } \\
27.5 \mathrm{~kW} \\
\text { Heating: } \\
180 \mathrm{~kW} \\
\text { Cooling: } \\
5 \mathrm{~kW}\end{array}$ & $\begin{array}{l}\text { Not } \\
\text { specified }\end{array}$ & ORC & $A C$ & $\begin{array}{l}\text { Calculations } \\
\text { for a } \\
\text { supermarket } \\
\text { in Vitoria, } \\
\text { Spain, } \\
\text { based on } \\
\text { experiments }\end{array}$ & $\begin{array}{l}\text { Based on a commercial ORC unit } \\
\text { by Rank }{ }^{\circledR} \text { with HFC-245fa as } \\
\text { working fluid } \\
\mathrm{CO}_{2} \text {-emissions savings: } 285 \text { t/y }\end{array}$ \\
\hline [106] & $\begin{array}{l}\text { Restaurant } \\
\text { case demand: } \\
\text { Electricity: } \\
35 \mathrm{~kW} \\
\text { Hot water: } \\
50 \mathrm{~kW} \\
\text { Cooling: } \\
10 \mathrm{~kW} \\
\text { Orange } \\
\text { processing } \\
\text { case demand: } \\
\text { Electricity: } \\
110 \mathrm{~kW} \\
\text { Hot water: } \\
115 \mathrm{~kW} \\
\text { Cooling: } \\
135 \mathrm{~kW}\end{array}$ & $\begin{array}{l}\text { Wood- } \\
\text { beech } \\
\text { (direct } \\
\text { combustion) }\end{array}$ & $\begin{array}{l}\text { Two ORC- } \\
\text { expanders }\end{array}$ & $\begin{array}{l}\text { Ammonia- } \\
\text { water AC }\end{array}$ & $\begin{array}{l}\text { RefProp } 9.0 \\
\text { model }\end{array}$ & $\begin{array}{l}\text { R134a and R407f selected as } \\
\text { working fluids } \\
\text { Trigeneration system with two } \\
\text { expanders and two compressors } \\
\text { allows for more flexible control of } \\
\text { thermal and electric services } \\
\text { The configuration is profitable in the } \\
\text { smaller restaurant case, but } \\
\text { unprofitable for the larger orange } \\
\text { processing case }\end{array}$ \\
\hline
\end{tabular}


Table 10 Cases of small-scale biomass-fired CCHP systems with ORCs with 50-400 kW nominal power

\begin{tabular}{|c|c|c|c|c|c|c|}
\hline Ref. & $\begin{array}{c}\text { Max. } \\
\text { Outputs }\end{array}$ & Biomass & $\begin{array}{l}\text { Prime } \\
\text { mover }\end{array}$ & Refrigeration & $\begin{array}{l}\text { Software/ } \\
\text { Location }\end{array}$ & Key Findings \\
\hline [107] & $\begin{array}{l}\text { Electricity: } \\
204 \mathrm{~kW} \\
\text { Heating: } \\
972 \mathrm{~kW} \\
\text { Cooling: } \\
226 \mathrm{~kW}\end{array}$ & $\begin{array}{l}\text { Direct } \\
\text { combustion: } \\
\text { - Willow } \\
\text { chips } \\
\text { - Straw } \\
\text { - Rice } \\
\text { husks }\end{array}$ & $\begin{array}{l}\text { ORC Turbine } \\
\text { (R245fa) }\end{array}$ & $A C$ & $\begin{array}{l}\text { Eclipse } \\
\text { Model }\end{array}$ & $\begin{array}{l}\text { Comparing three different biomass } \\
\text { inputs with the result that all three } \\
\text { fuels are feasible } \\
\text { Cogeneration has higher efficiency } \\
\text { than trigeneration } \\
\text { Payback period of trigeneration } \\
\text { slightly lower than for cogeneration } \\
\text { but much lower than for only } \\
\text { power } \\
\text { Fuel cost is an important factor } \\
\text { Similar study with ICE engine is } \\
\text { shown in Table } 2\end{array}$ \\
\hline [108] & $\begin{array}{l}\text { Electricity: } \\
280 \mathrm{~kW} \\
\\
\text { Heating: } \\
1,500 \mathrm{~kW} \\
\text { Cooling: } \\
500 \mathrm{~kW}\end{array}$ & $\begin{array}{l}\text { Combustion: } \\
\text { Oil tree } \\
\text { residues }\end{array}$ & $\begin{array}{l}\text { ORC } \\
\text { (fluid: } M D M \text { ) }\end{array}$ & $A C$ & $\begin{array}{l}\text { Model for an } \\
\text { airport in } \\
\text { Bari, Italy }\end{array}$ & $\begin{array}{l}\text { Energetic efficiency for heat and } \\
\text { power: } 71.8 \% \\
\text { Energetic efficiency for cooling } \\
\text { and power: } 31.2 \% \\
\text { Payback period of } 6 \text { years } \\
\text { Yearly } \mathrm{CO}_{2} \text {-savings: } 1,176 \text { t/year }\end{array}$ \\
\hline [109] & $\begin{array}{l}\text { Electricity: } \\
315 \mathrm{~kW} \\
\\
\text { Heating: } \\
\text { not specified } \\
\text { Cooling: } \\
780 \mathrm{~kW}\end{array}$ & $\begin{array}{l}\text { Pine Chips } \\
\text { (direct } \\
\text { combustion) }\end{array}$ & ORC & $A C$ & $\begin{array}{l}\text { SimaPro7.1 } \\
\text { Model for } \\
\text { the WALQA } \\
\text { Technology } \\
\text { park in } \\
\text { Huesca }\end{array}$ & $\begin{array}{l}\text { Energetic, economic and } \\
\text { environmental investigation of } \\
\text { different trigeneration } \\
\text { configurations: } \\
\text { - Parallel (boiler connected to ORC, } \\
\text { AC and heat-exchanger) } \\
\text { - Cascade (boiler only connected to } \\
\text { ORC) } \\
\text { - Separate energy service } \\
\text { production } \\
\begin{array}{l}\text { Cascade configuration leads to } \\
\text { highest energetic efficiencies. }\end{array} \\
\begin{array}{l}\text { Using the Life Cycle Assessment } \\
\text { methodology for environmental } \\
\text { impact }\end{array}\end{array}$ \\
\hline [110] & $\begin{array}{l}\text { Electricity: } \\
350 \mathrm{~kW} \\
\text { Heating: } \\
1,644 \mathrm{~kW} \\
\\
\text { Hot Water: } \\
0.52 \mathrm{~kg} / \mathrm{h} \\
\text { Cooling: } \\
2,000 \mathrm{~kW} \\
\text { Hydrogen: } \\
1.2-1.5 \mathrm{~kg} / \mathrm{h} \\
\text { Fresh Water: } \\
1-1.4 \mathrm{~kg} / \mathrm{h}\end{array}$ & $\begin{array}{l}\text { Pine Saw } \\
\text { Dust } \\
(0.2-0.4 \mathrm{~kg} / \mathrm{s} \\
- \text { direct } \\
\text { combustion) }\end{array}$ & ORC Turbine & $\begin{array}{l}\text { Double-effect } \\
\text { AC }\end{array}$ & $\begin{array}{l}\text { Model using } \\
\text { genetic } \\
\text { algorithm } \\
\text { optimization }\end{array}$ & $\begin{array}{l}\text { Exergetic efficiency: } \\
32-34 \% \\
\text { Increase in biomass flow -> } \\
\text { decrease in exergy efficiency }\end{array}$ \\
\hline
\end{tabular}


Table 11 Cases of small-scale biomass-fired and solar-assisted CCHP systems with ORCs with 400-1000 kW nominal power

\begin{tabular}{|c|c|c|c|c|c|c|}
\hline Ref. & $\begin{array}{c}\text { Max. } \\
\text { Outputs }\end{array}$ & Biomass & $\begin{array}{l}\text { Prime } \\
\text { mover }\end{array}$ & Refrigeration & $\begin{array}{l}\text { Software/ } \\
\text { Location }\end{array}$ & Key Findings \\
\hline [112] & $\begin{array}{l}\text { Electricity: } \\
600 \mathrm{~kW} \\
\text { Heating: } \\
3,750 \mathrm{~kW} \\
\text { Cooling: } \\
300 \mathrm{~kW}\end{array}$ & $\begin{array}{l}\text { Pine Saw } \\
\text { Dust } \\
\text { (direct } \\
\text { combustion) }\end{array}$ & $\begin{array}{l}\text { ORC } \\
\text { turbine }\end{array}$ & $\begin{array}{l}\text { Single-effect } \\
A C\end{array}$ & $\begin{array}{l}\text { Proposed } \\
\text { System } \\
\text { modelled in } \\
\text { EES }\end{array}$ & $\begin{array}{l}\text { Most exergy destroyed in biomass } \\
\text { burner (55\%) and ORC evaporator } \\
\text { (38\%) } \\
\text { System sensitive to ORC evaporator } \\
\text { temperature and pump inlet } \\
\text { temperature } \\
\text { System insensitive to turbine inlet } \\
\text { pressure } \\
\text { Maximum trigeneration exergy } \\
\text { efficiency } 28 \% \\
\text { Maximum trigeneration energetic } \\
\text { efficiency: } 89 \%\end{array}$ \\
\hline [113] & $\begin{array}{l}\text { Electricity: } \\
671 \mathrm{~kW} \\
\text { Hot water: } \\
2,617 \mathrm{~kW} \\
\text { Cooling: } \\
611 \mathrm{~kW} \\
\\
\text { Hydrogen: } \\
3.14 \mathrm{~kg} / \text { day }\end{array}$ & $\begin{array}{l}\text { Pine } \\
\text { sawdust } \\
(0.3 \mathrm{~kg} / \mathrm{s}- \\
\text { direct } \\
\text { combustion) }\end{array}$ & $\begin{array}{l}\text { ORC } \\
\text { Turbine } \\
\text { (n-octane) }\end{array}$ & $L i B r-A C$ & $\begin{array}{l}\text { Model and } \\
\text { Simulation }\end{array}$ & $\begin{array}{l}\text { Exergetic efficiency: } 22.2 \% \\
\text { Exergy destruction highest in } \\
\text { combustor and ORC evaporator } \\
\text { System affected mostly by pinch } \\
\text { point temperature, ORC inlet } \\
\text { pressure and ORC inlet temperature }\end{array}$ \\
\hline $\begin{array}{l}{[114]} \\
{[115]}\end{array}$ & $\begin{array}{l}\text { Electricity: } \\
400-800 \mathrm{~kW} \\
\text { Heating: } \\
\text { not specified } \\
\text { Cooling: } \\
\text { not specified }\end{array}$ & $\begin{array}{l}\text { Pine } \\
\text { sawdust } \\
\text { (direct } \\
\text { combustion) }\end{array}$ & $\begin{array}{l}\text { ORC } \\
\text { Turbine (in } \\
\text { some } \\
\text { cases } \\
\text { after } \\
\text { SOFC) }\end{array}$ & $\begin{array}{l}\text { Single-effect } \\
A C\end{array}$ & $E E S$ & $\begin{array}{l}\text { Comparing three different } \\
\text { configurations: } \\
\text { - Methane-fueled SOFC before ORC } \\
\text { - Biomass-boiler before ORC } \\
\text { - Solar-thermal boiler before ORC } \\
\text { Electrical efficiency of SOFC-system } \\
\text { highest with 19\%, } \\
\begin{array}{l}\text { Trigeneration energetic efficiency } \\
\text { highest in biomass and solar system } \\
\text { with both 90\% }\end{array} \\
\begin{array}{l}\text { Solar trigeneration system performs } \\
\text { best in terms of thermoeconomics and } \\
\text { ecology }\end{array}\end{array}$ \\
\hline [117] & $\begin{array}{l}\text { Electricity: } \\
1000 \mathrm{~kW} \\
\text { Hot water: } \\
\text { not specified } \\
\text { Cooling: } \\
\text { not specified }\end{array}$ & $\begin{array}{l}\text { Mixture of } \\
\text { agricultural } \\
\text { and forest } \\
\text { biomass } \\
\text { (direct } \\
\text { combustion) }\end{array}$ & ORC & $\begin{array}{l}\text { AC (single- } \\
\text { effect) }\end{array}$ & $\begin{array}{l}\text { GIS-Data } \\
\text { from } \\
\text { BIORAISE }\end{array}$ & $\begin{array}{l}\text { Max. electrical efficiency: } 15 \% \\
\text { Max. thermal efficiency: } 74 \% \\
\text { Studying the potential of such an } \\
\text { ORC-system in various regions of } \\
\text { Spain for villages of around } 10,000 \\
\text { inhabitants } \\
\text { Apart from regional climate, fuel ability } \\
\text { dictates profitability } \\
\text { CCHP profitable in warm climates, } \\
\text { CHP in cold climates }\end{array}$ \\
\hline
\end{tabular}

\subsection{Steam and Gas Turbines}

Conventional steam and gas turbines can serve electric loads of several hundreds of MW, but internally fired gas turbines are rarely smaller than $500 \mathrm{~kW}$ while steam turbines are rarely smaller than $50 \mathrm{~kW}$ [21]. Both types of turbines suffer significant losses in efficiency when used in partial load mode, so steam and gas turbines are an inconvenient choice for flexible small-scale CCHP systems [26]. 
However, the high temperature of exhaust gases holds high potential for further usage for heating and cooling applications. Studies suggest that small-scale gas turbines with 100-600 kW electric output are economically disadvantageous compared to internal combustion gas engines, but future technologies could change this status [118]. A summary of the two previous cases is given in Table 12.

There are certain studies that investigate the use of considerably small steam and gas turbines in CCHP systems, providing useful insights for other small-scale systems, especially run with micro-turbines as a prime mover. It can be found that for smaller systems $(2-3.5 \mathrm{MW})$ the highest exergy losses occur in the furnace [119] and that the values of energy efficiency of steam and gas turbines are similar for CHP systems [120]. One case where a gas turbine cycle of unspecified nominal power is combined with two ORC cycles has been mentioned in chapter 4.4.

\subsection{Micro Turbines}

Micro-turbines are another promising technology on the brink of mass-scale commercialization for CHP and CCHP systems [121]. Micro-turbine sizes range from a few $\mathrm{kW}$ to several hundreds of $\mathrm{kW}$, while even smaller capacities are being investigated. Apart from the disadvantages mentioned in Table 1 , there is also the need for high quality fuels when run on bio- or syngas [21]. A solution to this problem could be externally fired micro-turbines, where studies suggest that syngas containing more tar can lead to higher electric efficiencies [122]. Simulations of a biomass CHP system comparing an internally fired micro-turbine fueled with syngas to an externally fired micro-turbine driven by direct combustion could show that the latter reaches higher electric efficiency and higher overall efficiency with less biomass consumption [123]. In a case study for a polygeneration system, a $100 \mathrm{~kW}$ micro turbine was driven externally using direct combustion of biomass and natural gas [124]. It could be shown that with a mixture of $70 / 30$ for bio/ natural gas fuel, an electric efficiency of $21.8 \%$ could be reached, but a further increase of biomass would lower efficiency. Two cases of a trigeneration system using a micro gas turbine are presented in Table 12. 
Table 12 Cases of small-scale biomass-fired CCHP systems with steam, gas and micro turbines

\begin{tabular}{|c|c|c|c|c|c|c|}
\hline Ref. & $\begin{array}{c}\text { Max. } \\
\text { Outputs }\end{array}$ & Biomass & $\begin{array}{l}\text { Prime } \\
\text { mover }\end{array}$ & Refrigeration & $\begin{array}{l}\text { Software/ } \\
\text { Location }\end{array}$ & Key Findings \\
\hline [125] & $\begin{array}{l}\text { Electricity: } \\
1.1 \mathrm{MW} \\
\text { Cooling: } \\
0.26 \mathrm{MW} \\
\text { Net utility } \\
\text { Heating: } \\
0.22 \mathrm{MW} \\
\text { Ethanol: } \\
3.38 \mathrm{t} / \mathrm{h}\end{array}$ & $\begin{array}{l}\text { Fluidized } \\
\text { Bed gasifier } \\
(1 \text { t/h): } \\
\text { - Rice straw } \\
\text { - Sugarcane } \\
\text { - Coconut } \\
\text { fibres }\end{array}$ & $\begin{array}{l}\text { Gas } \\
\text { Turbine } \\
+ \\
\text { Steam } \\
\text { Turbines }\end{array}$ & $\begin{array}{l}\text { Vapour } \\
\text { absorption } \\
\text { refrigeration }\end{array}$ & $\begin{array}{l}\text { Aspen } \\
\text { Plus }\end{array}$ & $\begin{array}{l}\text { Polygeneration system for Ethanol production } \\
\text { plant } \\
\text { Comparison of different Biomass inputs and } \\
\text { outputs: } \\
\text { 1. Rice straw } \\
\text { 2. Sugarcane bagasse } \\
\text { 3. Coconut fibre dust } \\
\text { (highest output) } \\
\text { Fuel energy saving ratio between } 16-27 \% \\
\text { Payback period: } 5.25 \text { years } \\
\text { Also } \mathrm{CO}_{2} \text { capture technologies proposed }\end{array}$ \\
\hline [126] & $\begin{array}{l}\text { Electricity: } \\
172.28 \\
k W \\
\text { Heating: } \\
488.51 \\
k W \\
\text { Cooling: } \\
256.21 \\
k W\end{array}$ & $\begin{array}{l}\text { Corn Stover } \\
\text { (gasified) } \\
+ \\
\text { Coal }\end{array}$ & $\begin{array}{l}\text { Two Gas } \\
\text { Turbines } \\
\text { in } \\
\text { cascade } \\
\text { utilization }\end{array}$ & $\begin{array}{l}\text { Double-effect } \\
\text { Li-Br AC } \\
\text { (COE:1.1) }\end{array}$ & $\begin{array}{l}\text { Aspen } \\
\text { Plus } \\
\text { Software }\end{array}$ & $\begin{array}{l}\text { Chemical looping of coal and biomass in the } \\
\text { same gasifier } \\
\text { When co-firing with a ratio of } 50 / 50 \text {, an } \\
\text { energy efficiency of } 60.16 \% \text { and } 57.46 \% \text { can } \\
\text { be reached in summer and winter, } \\
\text { respectively } \\
\text { An increase in biomass firing leads to a } \\
\text { decrease in energy output, but an increase in } \\
\text { PESR }\end{array}$ \\
\hline [127] & $\begin{array}{l}\text { For one } \\
\text { MGT: } \\
\text { Electricity: } \\
30 \mathrm{~kW} \\
\text { Heating: } \\
57 \mathrm{~kW} \\
\text { Cooling: } \\
40 \mathrm{~kW}\end{array}$ & $\begin{array}{l}\text { Biogas } \\
\text { (supported } \\
\text { by natural } \\
\text { gas) }\end{array}$ & $\begin{array}{l}\text { Micro } \\
\text { Gas } \\
\text { Turbine }\end{array}$ & $\begin{array}{l}\text { Single and } \\
\text { double-effect } \\
\text { AC, } \\
\text { Ammonia- } \\
\text { water chiller } \\
\text { Compression } \\
\text { chiller }\end{array}$ & $\begin{array}{l}\text { Hysys } \\
\text { Process } \\
\text { Software }\end{array}$ & $\begin{array}{l}\text { Case study for sewage treatment plant with } \\
\text { comparison of several configurations using } \\
\text { various refrigeration technologies } \\
\text { Double-effect AC best economic and } \\
\text { energetic performance } \\
\text { Several micro turbines can be stacked for } \\
\text { higher electricity generation }\end{array}$ \\
\hline [128] & $\begin{array}{l}\text { Electricity: } \\
100 \mathrm{~kW} \\
\text { Heating: } \\
333 \mathrm{~kW} \\
\text { Cooling: } \\
110 \mathrm{~kW}\end{array}$ & $\begin{array}{l}\text { Not } \\
\text { mentioned } \\
\text { (Co-firing } \\
\text { natural gas } \\
\text { - direct } \\
\text { combustion) }\end{array}$ & $\begin{array}{l}\text { Micro } \\
\text { turbine }\end{array}$ & $\begin{array}{l}\mathrm{LiBr}-\mathrm{H} 2 \mathrm{O} \\
\text { single-effect } \\
A C\end{array}$ & $\begin{array}{l}\text { Gate- } \\
\text { Cycle }{ }^{8}\end{array}$ & $\begin{array}{l}\text { Thermal efficiency } 46-38 \% \\
\text { Electric efficiency } 30-19 \% \\
\text { Global energy efficiency of } 30-40 \% \\
\text { unsatisfactory for Italian high efficiency } \\
\text { system requirements } \\
\text { Trigeneration only viable in high-cooling } \\
\text { demand areas (hot areas) } \\
\text { Mix of } 50 / 50 \text { biomass gas/natural gas shows } \\
\text { best results considering energy conversion } \\
\text { efficiency, investment and fuel costs }\end{array}$ \\
\hline [129] & $\begin{array}{l}\text { Electricity: } \\
131 \mathrm{~kW} \\
\text { Heating: } \\
230 \mathrm{~kW} \\
\text { Cooling: } \\
187 \mathrm{~kW}\end{array}$ & $\begin{array}{l}\text { Corn Stover } \\
\text { gasified with } \\
\text { coal }\end{array}$ & $\begin{array}{l}\text { Steam } \\
\text { turbine } \\
\text { after } \\
\text { gasifier } \\
\text { and gas } \\
\text { turbine } \\
\text { using } \\
\text { syngas }\end{array}$ & $\begin{array}{l}\text { Double-effect } \\
\text { LiBr-AC }\end{array}$ & $\begin{array}{l}\text { Aspen } \\
\text { Plus }\end{array}$ & $\begin{array}{l}\text { Energy efficiency (winter/summer): } 63 \% / 67 \% \\
\text { Exergy efficiency (winter/summer): } 26 \% / 24 \% \\
\text { Increasing the share of biomass from } 0 \text { to } \\
50 \% \text { leads to efficiency losses of around } \\
25 \%, \text { but leads to } \mathrm{CO}_{2} \text { reduction of } 300 \% \text { by } \\
\text { using carbon capture technology }\end{array}$ \\
\hline
\end{tabular}




\subsection{Solar Extensions}

In comparison to bioenergy, solar energy has an even bigger potential with negligible operational costs as there are no fuel costs [130]. With the costs for PV cells plummeting from $\$ 76.67$ per Watt in 1977 to \$0.36 per Watt in 2014 and the use of solar thermal energy always having been simple and cheap, solar energy becomes more and more competitive [17]. The key findings and characteristics of a solar-driven CCHP system with an auxiliary biomass boiler are shown in Table 13. Another study considering a purely solar-driven CCHP system based on an ORC was mentioned in Table 11.

Table 13 Cases of small-scale solar-driven CCHP systems with auxiliary biomass boiler

\begin{tabular}{|c|c|c|c|c|c|c|}
\hline Ref. & $\begin{array}{c}\text { Max. } \\
\text { Outputs }\end{array}$ & Biomass & $\begin{array}{l}\text { Prime } \\
\text { mover }\end{array}$ & Refrigeration & $\begin{array}{l}\text { Software/ } \\
\text { Location }\end{array}$ & Key Findings \\
\hline $\begin{array}{l}{[16]} \\
{[131]}\end{array}$ & $\begin{array}{l}\text { Electricity: } \\
\text { variable with } \\
\text { solar input } \\
\text { Heating: } \\
\text { variable with } \\
\text { solar input } \\
\text { Cooling: } \\
\text { variable with } \\
\text { solar input } \\
\text { Potable water }\end{array}$ & $\begin{array}{l}\text { Wood chips } \\
\text { (direct } \\
\text { combustion) }\end{array}$ & $\begin{array}{c}\text { Solar } \\
\text { thermic and } \\
P V \text { system } \\
\text { with } \\
\text { auxiliary } \\
\text { biomass } \\
\text { boiler }\end{array}$ & $\begin{array}{c}\mathrm{LiBr}-\mathrm{H}_{2} \mathrm{O} \\
\mathrm{AC}\end{array}$ & $\begin{array}{l}\text { TRNSYS } \\
\text { for a } \\
\text { system } \\
\text { placed in } \\
\text { Naples, } \\
\text { Italy }\end{array}$ & $\begin{array}{c}\text { Electric Efficiency: } \\
\text { up to } 23 \% \\
\text { Thermal Efficiency: } \\
\text { up to } 60 \% \\
\text { Payback time of } 13.6 \text { years saving } \\
0.26 \text { million €/year } \\
\text { Very effective in summer, but very } \\
\text { ineffective in winter } \\
\text { The models prove that such a system } \\
\text { is not economically sensible except } \\
\text { on disadvantaged areas like small } \\
\text { islands }\end{array}$ \\
\hline
\end{tabular}

In direct comparison with bioenergy or fossil fuels, solar energy is disqualified for fueling CHP or CCHP systems on its own [114]. It is therefore necessary to combine solar energy with either advanced electric and/ or thermal storages or with other energy sources like biofuels [17,20]. Using solar energy this way in a CCHP system can lead to significant reductions of $\mathrm{CO}_{2}$ emissions and to higher energy utilization compared to stand-alone as well as CHP systems [132]. For solar extensions in bio-driven small-scale CCHP systems, there are five main configurations in which the solar energy can enhance the performance of the whole system (as illustrated in Figure 2):

1. Solar thermal energy can enhance the biochemical or thermochemical conversion of biomass into fuel for the prime mover to reduce biomass consumption (example case [133]).

2. Solar thermal energy can be used to preheat the air intake of the prime mover, which then will increase the efficiency of a combustion process (example case [134]). This is quite common for gas turbines and bigger systems.

3. Solar thermal energy can be used to produce steam directly and to temporarily run the prime mover without any usage of biomass (example case [114,133]). This configuration is often applied in systems using ORCs.

4. Solar thermal energy can be used to reheat any stream after the exhaust for enhancing the heat supply or the thermally activated cooling process. This way, when electricity demand is low, the heat and cooling demand can still be satisfied in partial mode and biomass can be saved (example case [83]).

5. PV cells can provide electric energy and thus the prime mover has to satisfy less electric demand, so that biomass can be saved (example case [135]). 


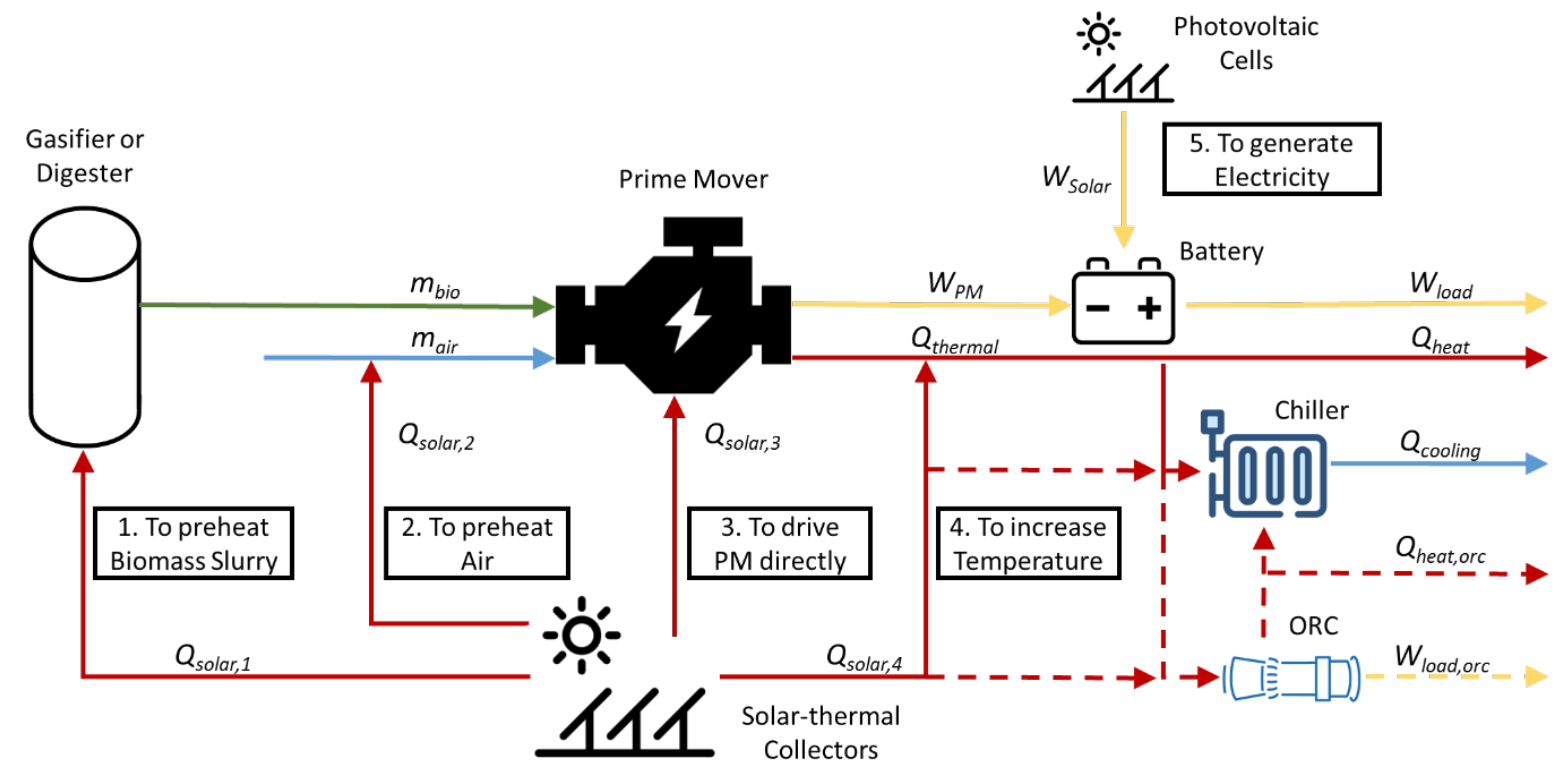

Figure 2 Possible solar extensions in biomass-fired small-scale CCHP systems

This figure does not represent PV/ thermal (PVT) components, which are essentially a combination of PV and solar thermal technology. Such PVT collectors are still not reliable and cost-effective enough for market penetration, but are promised to have huge potential in the near future [136]. As for Figure 2, the application of PVT collectors in a small-scale CCHP system would be a combination of any of the options 1-4 with option 5.

Essentially all five configurations imply a temporary decrease in biomass consumption. This has several advantages for the system:

- Operational costs can be lowered as there will be less costs for the biomass itself and the biomass transport to the system [24].

- It leads to a better short-term $\mathrm{CO}_{2}$ balance, because the combusted biomass needs several years to be reincorporated into the biomass cycle, as well as to a better long-term $\mathrm{CO}_{2}$ balance, because less energy will be used in transport vehicles.

- In some cases, the possibility to run systems only on solar energy can be a fallback option for stand-alone systems in case of a shortage of biomass supply.

However, there are also certain drawbacks to be considered:

- The installation of additional solar units leads to higher capital costs.

- The installation of additional solar units leads to higher complexity for the design and operation of the CCHP.

\section{Cooling Units and Storage Systems}

Seizing the exhaust heat from a prime mover by connecting it with a cooling unit can lead to higher profitability [137]. Similarly energy storage technologies improve the economic viability of small-scale CCHP systems [138]. It is therefore imperative for a clear picture of small-scale CCHP systems to know about the most important thermally activated cooling technologies and storage technologies.

\subsection{Thermally Activated Cooling Technologies}

Although CHP systems have been in use since the installation of the first commercial power plant, CCHP systems have only experienced very slow development until the oil crisis in 1979 [139]. Since then the commercialisation of thermally activated cooling units accelerated and with it the development of CCHP systems. A commonly used parameter to describe the efficiency of refrigeration units is the coefficient of performance (COP), which is the cooling capacity of the unit divided by its heat or electricity consumption [74]. 
Three different thermal refrigeration technologies can be applied in CCHP systems [140]:

- Absorption chillers (ACs)

- Adsorption chillers

- Desiccant chillers

ACs are the most commonly used type of thermally activated chillers, especially Lithium BromideWater ( $\mathrm{LiBr}$ ) and Water-Ammonia chillers [12,20,140]. In LiBr-chillers evaporating a low pressure, working fluid (water) in an evaporator "draws" heat and hence cools the surrounding. The gaseous water is then absorbed by the LiBr-salt solution in the absorber, the mixture reheated to evaporate the water and the water then condensed. The process scheme of a single-effect absorption refrigerator is illustrated in Figure 3.

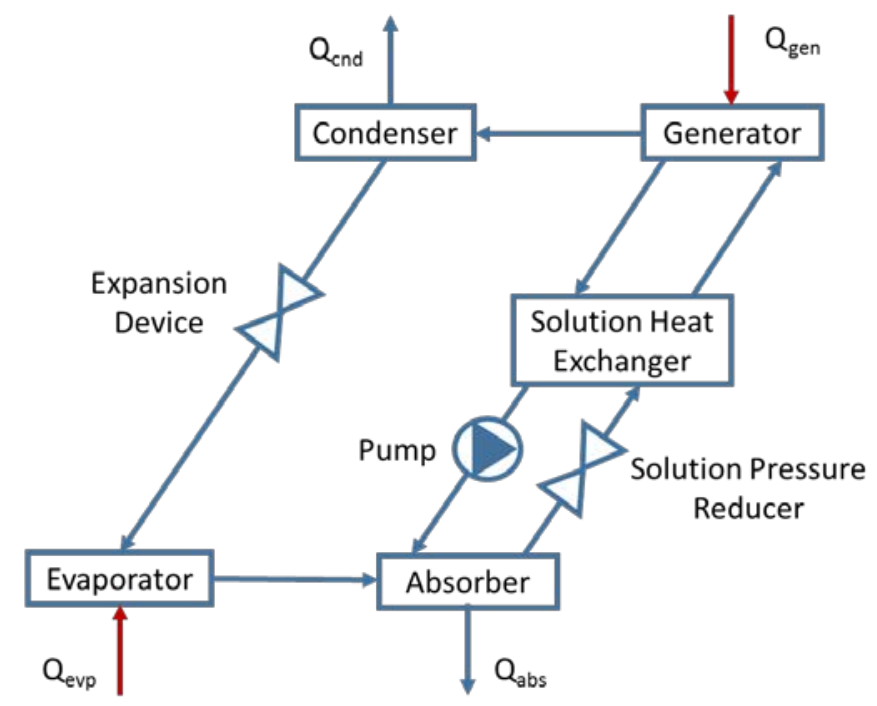

Figure 3 Simplified process of Single-Effect Absorption Refrigeration (based on [149])

$\mathrm{LiBr}$-ACs can be driven by pressurized hot water with more than $120^{\circ} \mathrm{C}$ or gases with more than $400^{\circ} \mathrm{C}$. Their advantages are the familiarity with the technology, the low investment costs and the high COP. Ammonia-water chillers work under the same basic principle as LiBr-chillers, but due to the lower evaporation temperature of ammonia, they can be used for lower temperature applications and in smaller sizes [12,139,140].

Another technology, which can be applied to low-heat sources and which is more environmentally friendly, is adsorption chilling. The basic working principle is similar ACs; however, the working fluid is only adsorbed to the surface of the adsorbent. The most common adsorption chillers use silica gel, as this type has been successfully commercialized [140]. Drawbacks of adsorption chillers are higher investment costs [12] and lower COP [139]. Nonetheless, they are sometimes chosen for small-scale CCHP systems (see Table 4, Table 7 and Figure 4).

A novel technology emerging on the market is desiccant cooling, where either a desiccant wheel or a liquid desiccant dries and heats up an incoming air stream, which can then be cooled down by surrounding air. In an evaporator the air then is rehumidified while its temperature is lowered immensely [141]. Desiccant cooling can be advantageous in small-scale residential systems due to its low maintenance costs and combined control of humidity and temperature [140]. Two cases using desiccant cooling could be found (see Table 6 and Table 9). Apart from thermally driven refrigeration systems, vapour compression chillers driven by electric or mechanical energy are often chosen in CCHP systems [5]. The advantages of vapour compression chillers are high COP and higher reliability due to independence from the thermal heat of the system, but they are less environmentally friendly, emit more noise and require more maintenance [2,32]. 


\subsection{Storage Units}

In the context of solar energy, batteries are often identified as the means to enable versatility of solar energy sources, hence making solar energy a feasible alternative to conventional energy sources [142]. The worldwide energy capacity of batteries for utility-scale energy storage applications is predicted to rise from $412 \mathrm{MWh}$ in 2014 to 51,200 MWh in 2023, which implies an annual growth rate of more than $71 \%$ [143]. A plurality of battery technologies is currently investigated and a review of all of these is beyond the scope of this paper. Hence, it is here referred to recent reviews of battery technologies for further information $[144,145]$.

Concerning thermal storages, simple water tanks are the most common and simplest solution, as water has a high specific capacity and the installation of a water tank is an easy and cheap task. A more compact way to store thermal energy can be provided by phase change materials (PCM). These materials use the energy needed for a phase change to either store or absorb heat [146]. Apart from storing energy for direct heating or cooling purposes, the heat can also be stored to preheat biofuels at a constant temperature and hence help with the start-up of an engine as well as to increase its efficiency [147].

\section{Results and Discussion}

As can be seen in Figure 4, most studies were simulation models, which themselves were often based on synthetic data or data collected from subsystems. Although there are some experimental studies, it seems that there is a general lack of real-life data in this area [148]. This may be due to an underestimation from researches of increased maintenance works and complexity, as operators may value the simplicity of a system highly. Additionally, as with every new technology, the overall lifetime of the components as well as their decay over time cannot be determined based on operation experience. There might be more real-life examples outside of scientific literature whose insights have not been documented for the scientific community. It was also found that nearly one out of four cases considers solar-assistance to enhance the biomass-fired systems. The potential of combining these two energy sources has thus been recognised.

In the cases of biomass-fired CCHP systems investigated in this paper, downdraft gasifiers seem to be the most practical choice for gasification due to the high quality of the syngas [40]. These gasification systems are mostly below $500 \mathrm{~kW}$ nominal power, as smaller gasification systems are generally more cost-effective than larger ones due to lower maintenance costs [10]. In the case studies investigated in this paper, anaerobic digestion is also an often-used technology. While for off-grid CCHP systems thermal and electric storage units are usually indispensable, for on-grid systems they are often overlooked in models despite their potentially high impact on economics [18]. If the use of a thermal storage system was stated at all, it was usually water tanks and only in one case PCM.

It could be found that LiBr-absorption is the most often used technology in the investigated cases of biomass-fired and solar-assisted CCHP systems. Ammonia-water chillers have also been proposed often, while for some models the concrete working pair for the $\mathrm{AC}$ has not been specified. As shown in Figure 4, AC technology is used significantly more often than other technologies. 


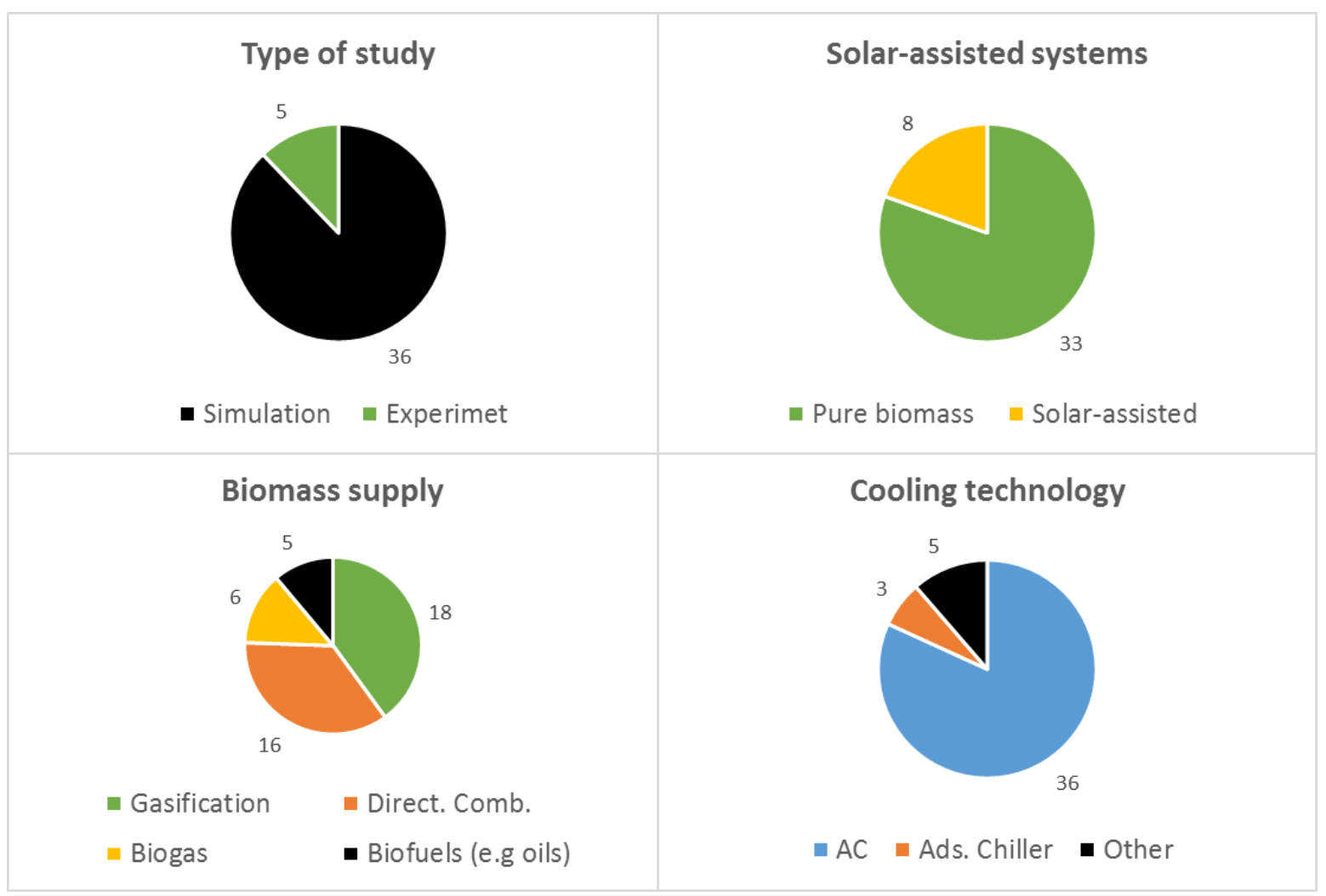

Figure 4 Meta-data results of the 41 different systems studied (multiple component application possible)

Payback time was chosen as the most convenient economic variable, but it is difficult to compare the economic viability of different systems based on this variable, because some studies include subsidies while some do not. It is remarkable that almost every model achieved economic viability, at least when subsidised. Together with the lack of studies on real-life, successful systems, this suggests a certain economic bias within the scientific community towards such renewable energy systems and their efficiencies (see also [149,150]). To promote small-scale biomass systems in rural and off-grid areas, where the benefits may be strongest, it is necessary to inform the population about the benefits and to educate the system owner and operators in technical training institutes [151].

Most case studies identify the prime mover as the most important unit of the CCHP system, because it has the biggest impact on energetic, ecologic and economic performance. Future research in the fields of SEs, FCs and micro turbines can lead to considerable competitiveness of these technologies compared to ICEs, while ORCs are already penetrating the market successfully $[12,21,25,48]$. Despite of the instability with bio- and syngas and the regular maintenance requirements of ICEs, until today they still seem to be the preferred choice for investors. But due to the external combustion SEs or Micro turbines can be used in combination with gasification for greater robustness [89,122]. Additionally, these technologies could surpass the performance of ICE driven systems and can lead to small-scale CCHP systems with even higher ecologic and economic benefits. However, other technologies concerning heat exchangers, cooling units, gasifiers or digesters will also develop further due to new materials and better simulation programs [18,152-155].

This study concentrated on systems which produce electricity, heating and cooling, but there are several polygeneration systems which produce other energy products like biofuels (e.g. hydrogen, methane), chemicals for production processes, purified water or food [156,157]. These technologies offer a huge variety of different system configurations for more efficient, more flexible and more environmentally friendly biomass usage, but they also increase the complexity of such systems. While some studies explicitly concentrate on storage possibilities, especially for off-grid systems, other studies completely ignore the possible benefits of storing electric and/ or thermal energy. Nonetheless, on- 
going advances in fields like phase-change materials or solid-state batteries will enter the energy market and increase the economic feasibility of small-scale CCHP systems, especially for off-grid systems and for solar-assisted systems [138,158-160]. For grid-connected systems data optimization, which will enable better synchronization of various interconnected CCHP systems, and load shifting techniques will improve their energetic and economic efficiency [138,161,162]. Other promising technologies emerging on the market are carbon capture and storage (CCS), where the $\mathrm{CO}_{2}$ is stored away, and carbon capture and utilisation (CCU), where the $\mathrm{CO}_{2}$ is used for chemical products. CCS and CCU can push the carbon balance of biomass-fired CCHP systems into negative areas [5,163].

Overall, the CCHP market is currently experiencing enormous growth and several technologies are competing with each other while also opening up new possibilities [14,21]. The same can be said for the research in various subfields; several technologies now passed the experimental stage and can be applied and investigated in CCHP systems, while unripe technologies will enter this stage soon. Hence, the amount of studies in this field is growing rapidly [25].

\section{Conclusion}

In this study, the most important components for biomass-fired, small-scale CCHP systems have been presented and described according to their most current development. Furthermore, for each prime mover, the most recent case studies and their key characteristics have been presented. The results of these findings have been discussed and put into the context of current and future technology development.

Although the field of small-scale biomass-fired and biomass-fired solar-assisted CCHP systems gains rising interest in the scientific community, most studies are based on simulation models instead of experimental data. This leads to a gap between theoretical and practical feasibility of such systems. Models often show very cost-effective and feasible systems, but investors seem to be reluctant due to the uncertainty involved. This suggests an economic bias within the scientific field towards such systems. Future policy implementations should therefore consider the need for extra maintenance in combination with the increased complexity of such systems. Flexible and fast responding systems should be promoted. Especially regions with high energy prices, like islands or off-grid, rural areas, should be targeted for financial support. However, also in regions with good electric infrastructure, these small systems may help with the goal of decentralization and lead to higher energy resilience of communities and individuals. Research and finance projects should not just monitor the design and construction of such systems, but also observe long-term technical and socio-economic effects.

Additionally, it was found, that many studies consider ICEs and ORCs as prime mover, but only few investigate the possibilities of other upcoming prime mover technologies like SEs, FCs or micro turbines. Especially in the context of biomass gasification prime movers need to show robustness and fuel flexibility, which ICEs often lack. Therefore, the chances for other prime movers to gain greater shares in the market are very high. To accelerate this development, policy implementations should promote the use of alternative prime movers, especially in combination with innovative biomass pretreatment technologies. Governments could finance such projects directly or give incentives to companies investing in such technologies through tax reductions or establishing knowledge transfer networks.

Moreover, stricter execution of already established policies like $\mathrm{CO}_{2}$-taxes may accelerate the transition towards renewable CCHP systems. Nonetheless, all of the systems investigated require a stable supply of raw biomass or processed biofuels. Policy implementations should therefore consider that a higher biomass consumption for such systems may endanger entire ecosystems and may lead to competition with areas for food production. 


\section{Acknowledgements}

This research has been conducted in collaboration with UPC (Universitat Politècnica de Catalunya) and KTH Royal Institute of Technology, funded through Erasmus Mundus J oint Doctoral Programme SELECT+, the support of which is gratefully acknowledged. AI also acknowledges support from Spanish project MOET_BIA2016-77675-R.

\section{References}

[1] OECD Green Growth Studies Energy OECD Green Growth Studies 2011. https:/ / www.oecd.org/ greengrowth/ greening-energy/ 49157219.pdf (accessed August 22, 2017).

[2] Maraver D, Sin A, Sebastián F, Royo J . Environmental assessment of CCHP (combined cooling heating and power) systems based on biomass combustion in comparison to conventional generation. Energy 2013;57:17-23. doi:10.1016/j.energy.2013.02.014.

[3] Pöschl M, Ward S, Owende P. Evaluation of energy efficiency of various biogas production and utilization pathways. Appl Energy 2010;87:3305- 21. doi:10.1016/j.apenergy.2010.05.011.

[4] Alanne K, Saari A. Distributed energy generation and sustainable development. Renew Sustain Energy Rev 2006;10:539- 58. doi:10.1016/j.rser.2004.11.004.

[5] Murugan S, Horák B. Tri and polygeneration systems - A review. Renew Sustain Energy Rev 2016. doi:10.1016/j.rser.2016.01.127.

[6] Knowles J . Overview of small and micro combined heat and power (CHP) systems. Small Micro Comb. Heat Power Syst., 2011, p. 3- 16. doi:10.1533/ 9780857092755.1.3.

[7] Liu H. Biomass fuels for small and micro combined heat and power (CHP) systems: resources, conversion and applications. Small Micro Comb. Heat Power Syst., 2011, p. 88- 122. doi:10.1533/9780857092755.1.88.

[8] Moriarty P, Honnery D. What is the global potential for renewable energy? Renew Sustain Energy Rev 2012;16:244- 52. doi:10.1016/j.rser.2011.07.151.

[9] Maghanki MM, Ghobadian B, Najafi G, Galogah RJ. Micro combined heat and power (MCHP) technologies and applications. Renew Sustain Energy Rev 2013;28:510- 24. doi:10.1016/j.rser.2013.07.053.

[10] Strzalka R, Schneider D, Eicker U. Current status of bioenergy technologies in Germany. Renew Sustain Energy Rev 2017;72:801-20. doi:10.1016/j.rser.2017.01.091.

[11] Souza GM, Ballester MVR, de Brito Cruz CH, Chum H, Dale B, Dale VH, et al. The role of bioenergy in a climate-changing world. Environ Dev 2017. doi:10.1016/j.envdev.2017.02.008.

[12] Maraver D, Sin A, Royo J, Sebastián F. Assessment of CCHP systems based on biomass combustion for small-scale applications through a review of the technology and analysis of energy efficiency parameters. Appl Energy 2013;102:1303- 13. doi:10.1016/j.apenergy.2012.07.012.

[13] European Commission. Statistical Pocketbook. 2014. doi:10.2833/ 77358.

[14] Mao G, Zou H, Chen G, Du H, Zuo J. Past, current and future of biomass energy research: A bibliometric analysis. Renew Sustain Energy Rev 2015;52:1823- 33. doi:10.1016/j.rser.2015.07.141.

[15] Calise F, Cipollina A, Dentice d'Accadia M, Piacentino A. A novel renewable polygeneration system for a small Mediterranean volcanic island for the combined production of energy and water: Dynamic simulation and economic assessment. Appl Energy 2014;135:675- 93. doi:10.1016/j.apenergy.2014.03.064.

[16] Calise F, Dentice d'Accadia M, Piacentino A. Exergetic and exergoeconomic analysis of a renewable polygeneration system and viability study for small isolated communities. Energy 2015;92:290-307. doi:10.1016/j.energy.2015.03.056. 
[17] Kannan N, Vakeesan D. Solar energy for future world: - A review. Renew Sustain Energy Rev 2016;62:1092- 105. doi:10.1016/j.rser.2016.05.022.

[18] Sonar D, Soni SL, Sharma D. Micro-trigeneration for energy sustainability: Technologies, tools and trends. Appl Therm Eng 2014;71:790- 6. doi:10.1016/j.applthermaleng.2013.11.037.

[19] Adams TA, Ghouse J H. Polygeneration of fuels and chemicals. Curr Opin Chem Eng 2015;10:87-93. doi:10.1016/j.coche.2015.09.006.

[20] J radi M, Riffat S. Tri-generation systems: Energy policies, prime movers, cooling technologies, configurations and operation strategies. Renew Sustain Energy Rev 2014;32:396- 415. doi:10.1016/j.rser.2014.01.039.

[21] Liu M, Shi Y, Fang F. Combined cooling, heating and power systems: A survey. Renew Sustain Energy Rev 2014;35:1-22. doi:10.1016/j.rser.2014.03.054.

[22] Godefroy J , Boukhanouf R, Riffat S. Design, testing and mathematical modelling of a smallscale CHP and cooling system (small CHP-ejector trigeneration). Appl Therm Eng 2007;27:6877. doi:10.1016/j.applthermaleng.2006.04.029.

[23] Wang J-J , Xu Z-L, J in H-G, Shi G, Fu C, Yang K. Design optimization and analysis of a biomass gasification based BCHP system: A case study in Harbin, China. Renew Energy 2014; 71:572- 83. doi:10.1016/j.renene.2014.06.016.

[24] Sahoo U, Kumar R, Pant PC, Chaudhury R. Scope and sustainability of hybrid solar- biomass power plant with cooling, desalination in polygeneration process in India. Renew Sustain Energy Rev 2015;51:304- 16. doi:10.1016/j.rser.2015.06.004.

[25] Al-Sulaiman FA, Hamdullahpur F, Dincer I. Trigeneration: A comprehensive review based on prime movers. Int J Energy Res 2011;35:233-58. doi:10.1002/ er.1687.

[26] Wu DW, Wang RZ. Combined cooling, heating and power: A review. Prog Energy Combust Sci 2006;32:459-95. doi:10.1016/j.pecs.2006.02.001.

[27] Barbieri ES, Spina PR, Venturini M. Analysis of innovative micro-CHP systems to meet household energy demands. Appl Energy 2012;97:723- 33. doi:10.1016/j.apenergy.2011.11.081.

[28] Wang J -J , Zhang C-F, J ing Y-Y. Multi-criteria analysis of combined cooling, heating and power systems in different climate zones in China. Appl Energy 2010;87:1247- 59. doi:10.1016/j.apenergy.2009.06.027.

[29] European Commission. EU Reference Scenario 2016 Energy, transport and GHG emissions Trends to 2050 Main results. 2016.

[30] J oelsson J M, Gustavsson L. Reduction of $\mathrm{CO} 2$ emission and oil dependency with biomass-based polygeneration. Biomass and Bioenergy 2010;34:967- 84. doi:10.1016/j.biombioe.2010.02.005.

[31] Angrisani G, Akisawa A, Marrasso E, Roselli C, Sasso M. Performance assessment of cogeneration and trigeneration systems for small scale applications. Energy Convers Manag 2016;125:194- 208. doi:10.1016/j.enconman.2016.03.092.

[32] Angrisani G, Roselli C, Sasso M. Distributed microtrigeneration systems. Prog Energy Combust Sci 2012;38:502-21. doi:10.1016/j.pecs.2012.02.001.

[33] Yllmaz S, Selim H. A review on the methods for biomass to energy conversion systems design. Renew Sustain Energy Rev 2013;25:420- 30. doi:10.1016/j.rser.2013.05.015.

[34] Ali G, Ali A, Bashir MK, Ashfaq M, Ahmed A. Utilization of Rice Husk for Renewable Energy Potential in Pakistan: An economic perspective. Renew Sustain Energy Rev 2014;61:157- 60.

[35] Cutz L, Haro P, Santana D, J ohnsson F. Assessment of biomass energy sources and technologies: The case of Central America. Renew Sustain Energy Rev 2016;58. doi:10.1016/j.rser.2015.12.322.

[36] Li C, Aston J E, Lacey J A, Thompson VS, Thompson DN. Impact of feedstock quality and variation on biochemical and thermochemical conversion. Renew Sustain Energy Rev 2016;65:525-36. doi:10.1016/j.rser.2016.06.063. 
[37] Kumar A, Kumar N, Baredar P, Shukla A. A review on biomass energy resources, potential, conversion and policy in India. Renew Sustain Energy Rev 2015;45:530- 9.

doi:10.1016/j.rser.2015.02.007.

[38] Gebreegziabher T, Oyedun AO, Hui CW. Optimum biomass drying for combustion - A modeling approach. Energy 2013;53:67- 73. doi:10.1016/j.energy.2013.03.004.

[39] Chen Y, Yang H, Wang X, Zhang S, Chen H. Biomass-based pyrolytic polygeneration system on cotton stalk pyrolysis: Influence of temperature. Bioresour Technol 2012;107:411- 8. doi:10.1016/j.biortech.2011.10.074.

[40] Sansaniwal SK, Pal K, Rosen MA, Tyagi SK. Recent advances in the development of biomass gasification technology: A comprehensive review. Renew Sustain Energy Rev 2017; 72:363- 84. doi:10.1016/j.rser.2017.01.038.

[41] Laviola BG, Rodrigues EV, Teodoro PE, Peixoto L de A, Bhering LL. Biometric and biotechnology strategies in J atropha genetic breeding for biodiesel production. Renew Sustain Energy Rev 2017;76:894- 904. doi:10.1016/j.rser.2017.03.116.

[42] Gaurav N, Sivasankari S, Kiran G, Ninawe A, Selvin J. Utilization of bioresources for sustainable biofuels: A Review. Renew Sustain Energy Rev 2017;73:205- 14. doi:10.1016/j.rser.2017.01.070.

[43] Ramachandra TV, Durga Madhab M, Shilpi S, J oshi NV. Algal biofuel from urban wastewater in India: Scope and challenges. Renew Sustain Energy Rev 2013;21:767- 77. doi:10.1016/j.rser.2012.12.029.

[44] Qiu G, Liu H, Riffat S. Expanders for micro-CHP systems with organic Rankine cycle. Appl Therm Eng 2011;31:3301- 7. doi:10.1016/j.applthermaleng.2011.06.008.

[45] Al Moussawi H, Fardoun F, Louahlia-Gualous H. Review of tri-generation technologies: Design evaluation, optimization, decision-making, and selection approach. Energy Convers Manag 2016;120:157-96. doi:10.1016/j.enconman.2016.04.085.

[46] Aliabadi AA, Thomson MJ , Wallace J S, Tzanetakis T, Lamont W, Di Carlo J . Efficiency and Emissions Measurement of a Stirling-Engine-Based Residential Microcogeneration System Run on Diesel and Biodiesel. Energy \& Fuels 2009;23:1032-9. doi:10.1021/ ef800778g.

[47] Vélez F, SegoviaJJ , Martín MC, Antolín G, Chejne F, Quijano A. A technical, economical and market review of organic Rankine cycles for the conversion of low-grade heat for power generation. Renew Sustain Energy Rev 2012;16:4175- 89. doi:10.1016/j.rser.2012.03.022.

[48] Obernberger I, Carlsen H. State-of-the-art and future developments regarding small-scale biomass CHP systems with a special focus on ORC and Stirling engine technologies. Int. Nord. Bioenergy, 2003.

[49] Qian Y, Sun S, Ju D, Shan X, Lu X. Review of the state-of-the-art of biogas combustion mechanisms and applications in internal combustion engines. Renew Sustain Energy Rev 2017;69:50- 8. doi:10.1016/j.rser.2016.11.059.

[50] Pilavachi PA. Mini- and micro-gas turbines for combined heat and power. Appl Therm Eng 2002;22:2003- 14. doi:10.1016/S1359-4311(02)00132-1.

[51] He M, Zhang X, Zeng K, Gao K. A combined thermodynamic cycle used for waste heat recovery of internal combustion engine. Energy 2011;36:6821-9. doi:10.1016/j.energy.2011.10.014.

[52] Rovas D, Zabaniotou A. Exergy analysis of a small gasification-ICE integrated system for CHP production fueled with Mediterranean agro-food processing wastes: The SMARt-CHP. Renew Energy 2015;83:510- 7. doi:10.1016/j.renene.2015.04.071.

[53] Puig-Arnavat M, Bruno J C, Coronas A. Modeling of trigeneration configurations based on biomass gasification and comparison of performance. Appl Energy 2014;114:845- 56. doi:10.1016/j.apenergy.2013.09.013.

[54] Huang Y, Wang YD, Rezvani S, Mcllveen-Wright DR, Anderson M, Hewitt NJ . Biomass fuelled trigeneration system in selected buildings. Energy Convers Manag 2011;52:2448- 54. doi:10.1016/j.enconman.2010.12.053. 
[55] Wang Y, Huang Y, Roskilly AP, Ding Y, Hewitt N. Trigeneration running with raw jatropha oil. Fuel Process Technol 2010;91:348-53. doi:10.1016/j.fuproc.2009.11.006.

[56] Sturm B, Butcher M, Wang Y, Huang Y, Roskilly T. The feasibility of the sustainable energy supply from bio wastes for a small scale brewery - A case study. Appl Therm Eng 2012;39:4552. doi:10.1016/j.applthermaleng.2012.01.036.

[57] Wang J, Mao T, Sui J , J in H. Modeling and performance analysis of CCHP (combined cooling, heating and power) system based on co-firing of natural gas and biomass gasification gas. Energy 2015;93:801- 15. doi:10.1016/j.energy.2015.09.091.

[58] WangJ J , Mao T. Cost allocation and sensitivity analysis of multi-products from biomass gasification combined cooling heating and power system based on the exergoeconomic methodology. Energy Convers Manag 2015;105:230-9. doi:10.1016/j.enconman.2015.07.081.

[59] WangJ -J, Yang K, Xu Z-L, Fu C. Energy and exergy analyses of an integrated CCHP system with biomass air gasification. Appl Energy 2015;142:317-27. doi:10.1016/j.apenergy.2014.12.085.

[60] Gao P, Li W, Cheng Y, Tong Y, Dai Y, Wang R. Thermodynamic performance assessment of CCHP system driven by different composition gas. Appl Energy 2014;136:599- 610 . doi:10.1016/j.apenergy.2014.09.070.

[61] Gao P, Dai Y, Tong Y, Dong P. Energy matching and optimization analysis of waste to energy CCHP (combined cooling, heating and power) system with exergy and energy level. Energy 2015; 79:522- 35. doi:10.1016/j.energy.2014.11.050.

[62] Hossain AK, Thorpe R, Vasudevan P, Sen PK, Critoph RE, Davies PA. Omnigen: Providing electricity, food preparation, cold storage and pure water using a variety of local fuels. Renew Energy 2013;49:197-202. doi:10.1016/j.renene.2012.01.032.

[63] Wu D, Yu H, Harvey A, Roskilly AP. Micro distributed energy system driven with preheated Croton megalocarpus oil - A performance and particulate emission study. Appl Energy 2013;112:1383-92. doi:10.1016/j.apenergy.2013.04.071.

[64] Coronado CR, Yoshioka J T, Silveira J L. Electricity, hot water and cold water production from biomass. Energetic and economical analysis of the compact system of cogeneration run with woodgas from a small downdraft gasifier. Renew Energy 2011;36:1861- 8. doi:10.1016/j.renene.2010.11.021.

[65] Parise JAR, Castillo Martínez LC, Marques RP, Mena J B, Vargas J VC. A study of the thermodynamic performance and CO2 emissions of a vapour compression bio-trigeneration system. Appl Therm Eng 2011;31:1411-20. doi:10.1016/j.applthermaleng.2010.12.040.

[66] Li CY, Wu JY, Zheng CY, Wang RZ. Effect of LPG addition on a CCHP system based on different biomass-derived gases in cooling and power mode. Appl Therm Eng 2017;115:315- 25. doi:10.1016/j.applthermaleng.2016.12.120.

[67] Caliano M, Bianco N, Graditi G, Mongibello L. Analysis of a Biomass-fired CCHP System Considering Different Design Configurations. Energy Procedia 2017;105:1683- 91. doi:10.1016/j.egypro.2017.03.549.

[68] Caliano M, Bianco N, Graditi G, Mongibello L. Design optimization and sensitivity analysis of a biomass-fired combined cooling, heating and power system with thermal energy storage systems. Energy Convers Manag 2017;149:631-45. doi:10.1016/J .ENCONMAN.2017.07.048.

[69] Li CY, Wu JY, Zheng CY, Wang RZ. Effect of LPG addition on a CCHP system based on different biomass-derived gases in cooling and power mode. Appl Therm Eng 2017;115:315- 25. doi:10.1016/J .APPLTHERMALENG.2016.12.120.

[70] Li H, Zhang X, Liu L, Zeng R, Zhang G. Exergy and environmental assessments of a novel trigeneration system taking biomass and solar energy as co-feeds. Appl Therm Eng 2016;104:697- 706. doi:10.1016/j.applthermaleng.2016.05.081.

[71] Zhang X, Li H, Liu L, Zeng R, Zhang G. Analysis of a feasible trigeneration system taking solar energy and biomass as co-feeds. Energy Convers Manag 2016;122:74- 84. doi:10.1016/j.enconman.2016.05.063. 
[72] Stanek W, Gazda W, Kostowski W. Thermo-ecological assessment of CCHP (combined coldheat-and-power) plant supported with renewable energy. Energy 2015;92:279- 89. doi:10.1016/j.energy.2015.02.005.

[73] Gazda W, Stanek W. Energy and environmental assessment of integrated biogas trigeneration and photovoltaic plant as more sustainable industrial system. Appl Energy 2016;169:138-49. doi:10.1016/j.apenergy.2016.02.037.

[74] Wang J, Yang Y. Energy, exergy and environmental analysis of a hybrid combined cooling heating and power system utilizing biomass and solar energy. Energy Convers Manag 2016;124:566- 77. doi:10.1016/j.enconman.2016.07.059.

[75] Calise F, Palombo A, Vanoli L. Design and dynamic simulation of a novel polygeneration system fed by vegetable oil and by solar energy. Energy Convers Manag 2012;60:204-13. doi:10.1016/j.enconman.2012.02.014.

[76] Bang-Møller C, Rokni M, Elmegaard B, Ahrenfeldt J , Henriksen UB. Decentralized combined heat and power production by two-stage biomass gasification and solid oxide fuel cells. Energy 2013;58:527- 37. doi:10.1016/j.energy.2013.06.046.

[77] Samavati M, Raza R, Zhu B. Design of a 5-kW advanced fuel cell polygeneration system. Wiley Interdiscip Rev Energy Environ 2012;1:173- 80. doi:10.1002/ wene.6.

[78] Bocci E, Carlo A Di, McPhail SJJ , Gallucci K, Foscolo PUU, Moneti M, et al. Biomass to fuel cells state of the art: A review of the most innovative technology solutions. Int J Hydrogen Energy 2014;39:21876- 95. doi:10.1016/j.jjhydene.2014.09.022.

[79] Gong Y, Huang K. Study of a renewable biomass fueled SOFC: The effect of catalysts. Int J Hydrogen Energy 2013;38:16518- 23. doi:10.1016/j.jjhydene.2013.05.147.

[80] Abuadala A, Dincer I. Exergoeconomic analysis of a hybrid system based on steam biomass gasification products for hydrogen production. Int J Hydrogen Energy 2011;36:12780- 93. doi:10.1016/j.jjhydene.2011.07.067.

[81] Rahman SNA, Masdar MS, Rosli MI, Majlan EH, Husaini T, Kamarudin SK, et al. Overview biohydrogen technologies and application in fuel cell technology. Renew Sustain Energy Rev 2016;66:137- 62. doi:10.1016/j.rser.2016.07.047.

[82] Ozcan H, Dincer I. Performance evaluation of an SOFC based trigeneration system using various gaseous fuels from biomass gasification. Int J Hydrogen Energy 2015;40:7798- 807. doi:10.1016/j.jjhydene.2014.11.109.

[83] Ozcan H, Dincer I. Thermodynamic analysis of an integrated sofc, solar orc and absorption chiller for tri-generation applications. FUEL CELLS 2013;13:781-93. doi:10.1002/ fuce.201300012.

[84] Al-Sulaiman FA, Hamdullahpur F. Energy analysis of a trigeneration plant based on solid oxide fuel cell and organic Rankine cycle. Int J Hydrogen Energy 2010;35:5104- 13. doi:10.1016/j.ijhydene.2009.09.047.

[85] Al-Sulaiman FA, Dincer I, Hamdullahpur F. Exergy analysis of an integrated solid oxide fuel cell and organic Rankine cycle for cooling, heating and power production. J Power Sources 2010;195:2346- 54. doi:10.1016/j.jpowsour.2009.10.075.

[86] Palomba V, Prestipino M, Galvagno A. Tri-generation for industrial applications: Development of a simulation model for a gasification-SOFC based system. Int J Hydrogen Energy 2017;42:27866- 83. doi:10.1016/J .IJ HYDENE.2017.06.206.

[87] Al Moussawi H, Fardoun F, Louahlia H. Selection based on differences between cogeneration and trigeneration in various prime mover technologies. Renew Sustain Energy Rev 2017;74:491- 511. doi:10.1016/j.rser.2017.02.077.

[88] Harrod J, Mago PJ , Luck R. Sizing analysis of a combined cooling, heating, and power system for a small office building using a wood waste biomass-fired Stirling engine. Int J Energy Res 2012;36:64- 74. doi:10.1002/er.1782. 
[89] Renzi M, Brandoni C. Study and application of a regenerative Stirling cogeneration device based on biomass combustion. Appl Therm Eng 2014;67:341- 51. doi:10.1016/j.applthermaleng.2014.03.045.

[90] Balcombe P, Rigby D, Azapagic A. Environmental impacts of microgeneration: Integrating solar PV, Stirling engine CHP and battery storage. Appl Energy 2015;139:245- 59. doi:10.1016/j.apenergy.2014.11.034.

[91] Cotana F, Messineo A, Petrozzi A, Coccia V, Cavalaglio G, Aquino A. Comparison of ORC turbine and stirling engine to produce electricity from gasified poultry waste. Sustain 2014;6:5714- 29. doi:10.3390/su6095714.

[92] Kong XQ, Wang RZ, Huang XH. Energy efficiency and economic feasibility of CCHP driven by stirling engine. Energy Convers Manag 2004;45:1433- 42. doi:10.1016/j.enconman.2003.09.009.

[93] Arashnia I, Najafi G, Ghobadian B, Yusaf T, Mamat R, Kettner M. Development of Micro-scale Biomass-fuelled CHP System Using Stirling Engine. Energy Procedia 2015;75:1108- 13. doi:10.1016/j.egypro.2015.07.505.

[94] MITIE Group/ Waitrose. Biomass gasifier Stirling engine based tri-generation system in United Kingdom 2012:2. http:// biomasspower.gov.in/ document/ download-lef-tside/ International Case Study 1_31March.pdf (accessed March 24, 2017).

[95] Uzuneanu K, Scarpete D, Badea N. Thermoeconomical study of a prime mover Stirling engine in a micro CCHP biomass system for domestic residence. Adv ENERGY PLANNING, Environ Educ Renew ENERGY SOURCES Thermoeconomical 2010:75- 80.

[96] Uzuneanu+K, Scarpete+ D, Kamburova++ V. An assessment of commercial Stirling Engines for TRigeneration Systems. Termotehnica, 2016, p. 56-9.

[97] Scarpete D, Uzuneanu K, Badea C N. Stirling Engine in Residential Systems Based on Renewable Energy. Adv. ENERGY PLANNING, Environ. Educ. Renew. ENERGY SOURCES, 2010.

[98] Huang Y, Wang YD, Chen H, Zhang X, Mondol J, Shah N, et al. Performance analysis of biofuel fired trigeneration systems with energy storage for remote households. Appl Energy 2017;186:530- 8. doi:10.1016/j.apenergy.2016.03.028.

[99] Tchanche BF, Lambrinos G, Frangoudakis A, Papadakis G. Low-grade heat conversion into power using organic Rankine cycles - A review of various applications. Renew Sustain Energy Rev 2011;15:3963- 79. doi:10.1016/j.rser.2011.07.024.

[100] Liu H, Shao Y, Li J . A biomass-fired micro-scale CHP system with organic Rankine cycle (ORC) - Thermodynamic modelling studies. Biomass and Bioenergy 2011;35:3985- 94. doi:10.1016/j.biombioe.2011.06.025.

[101] Quoilin S, Broek M Van Den, Declaye S, Dewallef P, Lemort V. Techno-economic survey of Organic Rankine Cycle (ORC) systems. Renew Sustain Energy Rev 2013;22:168- 86. doi:10.1016/j.rser.2013.01.028.

[102] Saleh B, Koglbauer G, Wendland M, Fischer J . Working fluids for low-temperature organic Rankine cycles. Energy 2007;32:1210-21. doi:10.1016/j.energy.2006.07.001.

[103] J radi M, Riffat S. Experimental investigation of a biomass-fuelled micro-scale tri-generation system with an organic Rankine cycle and liquid desiccant cooling unit. Energy 2014;71:80-93. doi:10.1016/j.energy.2014.04.077.

[104] Karellas S, Braimakis K. Energy- exergy analysis and economic investigation of a cogeneration and trigeneration ORC- VCC hybrid system utilizing biomass fuel and solar power. Energy Convers Manag 2016;107:103-13. doi:10.1016/j.enconman.2015.06.080.

[105] Navarro-Esbrí J , Molés F, Peris B, Mota-Babiloni A, Pascual Martí J, Collado R, et al. Combined cold, heat and power system, based on an organic Rankine cycle, using biomass as renewable heat source for energy saving and emissions reduction in a supermarket. Energy Procedia 2017;129:652- 9. doi:10.1016/J .EGYPRO.2017.09.134. 
[106] Briola S, Di Marco P, Gabbrielli R. Thermodynamic sensitivity analysis of a novel trigeneration thermodynamic cycle with two-phase expanders and two-phase compressors. Energy 2017;127:335- 50. doi:10.1016/J.ENERGY.2017.03.135.

[107] Huang Y, Wang YD, Rezvani S, McIlveen-Wright DR, Anderson M, Mondol J , et al. A technoeconomic assessment of biomass fuelled trigeneration system integrated with organic Rankine cycle. Appl Therm Eng 2013;53:325- 31. doi:10.1016/j.applthermaleng.2012.03.041.

[108] Amirante R, Clodoveo ML, Distaso E, Ruggiero F, Tamburrano P. A tri-generation plant fuelled with olive tree pruning residues in Apulia: An energetic and economic analysis 2016. doi:10.1016/j.renene.2015.11.085.

[109] Maraver D, Rezeau ; A, Sebastián F, Royo J . Thermodynamic optimization of a trigeneration system based on biomass combustion. 17th Eur. biomass Conf. Exhib., Hamburg, Germany: 2009.

[110] Ahmadi P, Dincer I, Rosen MA. Thermoeconomic multi-objective optimization of a novel biomass-based integrated energy system. Energy 2014;68:958- 70. doi:10.1016/j.energy.2014.01.085.

[111] Al-Sulaiman FA, Dincer I, Hamdullahpur F. Energy and exergy analyses of a biomass trigeneration system using an organic Rankine cycle. Energy 2012;45:975- 85. doi:10.1016/j.energy.2012.06.060.

[112] Al-Sulaiman FA, Hamdullahpur F, Dincer I. Greenhouse gas emission and exergy assessments of an integrated organic Rankine cycle with a biomass combustor for combined cooling, heating and power production. Appl Therm Eng 2011;31:439- 46. doi:10.1016/j.applthermaleng.2010.09.019.

[113] Ahmadi P, Dincer I, Rosen MA. Development and assessment of an integrated biomass-based multi-generation energy system. Energy 2013;56:155-66. doi:10.1016/j.energy.2013.04.024.

[114] Al-Sulaiman FA, Hamdullahpur F, Dincer I. Performance comparison of three trigeneration systems using organic rankine cycles. Energy 2011;36:5741- 54 . doi:10.1016/j.energy.2011.06.003.

[115] Al-Sulaiman FA, Dincer I, Hamdullahpur F. Thermoeconomic optimization of three trigeneration systems using organic Rankine cycles: Part I - Formula. Energy Convers Manag 2013;69:199-208. doi:10.1016/j.enconman.2012.12.030.

[116] Al-Sulaiman FA, Dincer I, Hamdullahpur F. Thermoeconomic optimization of three trigeneration systems using organic Rankine cycles: Part II - Applications. Energy Convers Manag 2013;69:209- 16. doi:10.1016/j.enconman.2012.12.032.

[117] Uris M, Linares J I, Arenas E. Feasibility assessment of an Organic Rankine Cycle (ORC) cogeneration plant (CHP/CCHP) fueled by biomass for a district network in mainland Spain. Energy 2017;133:969- 85. doi:10.1016/J .ENERGY.2017.05.160.

[118] Arena U, Di Gregorio F, Santonastasi M. A techno-economic comparison between two design configurations for a small scale, biomass-to-energy gasification based system. Chem EngJ 2010;162:580-90. doi:10.1016/j.cej.2010.05.067.

[119] Lian ZT, Chua KJ , Chou SK. A thermoeconomic analysis of biomass energy for trigeneration. Appl Energy 2010;87:84-95. doi:10.1016/j.apenergy.2009.07.003.

[120] Bagdanavicius A, J enkins N, Hammond GP. Assessment of community energy supply systems using energy, exergy and exergoeconomic analysis. Energy 2012;45:247- 55. doi:10.1016/j.energy.2012.01.058.

[121] Pantaleo AM, Camporeale SM, Shah N. Thermo-economic assessment of externally fired microgas turbine fired by natural gas and biomass: Applications in Italy. Energy Convers Manag 2013;75:202-13. doi:10.1016/j.enconman.2013.06.017.

[122] Baina F, Malmquist A, Alejo L, Fransson TH. Effect of the fuel type on the performance of an externally fired micro gas turbine cycle. Appl Therm Eng 2015;87:150- 60. doi:10.1016/j.applthermaleng.2015.04.042. 
[123] Vera D, J urado F, de Mena B, Schories G. Comparison between externally fired gas turbine and gasifier-gas turbine system for the olive oil industry. Energy 2011;36:6720-30. doi:10.1016/j.energy.2011.10.036.

[124] Riccio G, Chiaramonti D. Design and simulation of a small polygeneration plant cofiring biomass and natural gas in a dual combustion micro gas turbine (BIO_MGT). Biomass and Bioenergy 2009;33:1520-31. doi:10.1016/j.biombioe.2009.07.021.

[125] J ana K, De S. Polygeneration using agricultural waste: Thermodynamic and economic feasibility study. Renew Energy 2015;74:648-60. doi:10.1016/j.renene.2014.08.078.

[126] Fan J , Hong H, Zhu L, J iang Q, J in H. Thermodynamic and environmental evaluation of biomass and coal co-fuelled gasification chemical looping combustion with $\mathrm{CO} 2$ capture for combined cooling, heating and power production. Appl Energy 2017;195:861- 76. doi:10.1016/j.apenergy.2017.03.093.

[127] Bruno J C, Ortega-López V, Coronas A. Integration of absorption cooling systems into micro gas turbine trigeneration systems using biogas: Case study of a sewage treatment plant. Appl Energy 2009;86:837-47. doi:10.1016/j.apenergy.2008.08.007.

[128] Pantaleo AM, Camporeale SM, Markides CN, Scarascia Mugnozza G, Shah N. Energy performance and thermo-economic assessment of a microturbine-based dual-fuel gas-biomass trigeneration system. Energy Procedia 2017;105:764- 72. doi:10.1016/j.egypro.2017.03.387.

[129] Fan J , Hong H, Zhu L, Jiang Q, J in H. Thermodynamic and environmental evaluation of biomass and coal co-fuelled gasification chemical looping combustion with $\mathrm{CO} 2$ capture for combined cooling, heating and power production. Appl Energy 2017;195:861- 76. doi:10.1016/J .APENERGY.2017.03.093.

[130] Panwar NL, Kaushik SC, Kothari S. Role of renewable energy sources in environmental protection: A review. Renew Sustain Energy Rev 2011;15:1513- 24. doi:10.1016/j.rser.2010.11.037.

[131] Calise F, Dentice d'Accadia M, Piacentino A. A novel solar trigeneration system integrating PVT (photovoltaic/thermal collectors) and SW (seawater) desalination: Dynamic simulation and economic assessment. Energy 2014;67:129-48. doi:10.1016/j.energy.2013.12.060.

[132] Nosrat AH, Swan LG, Pearce J M. Improved performance of hybrid photovoltaic-trigeneration systems over photovoltaic-cogen systems including effects of battery storage. Energy 2013;49:366- 74. doi:10.1016/j.energy.2012.11.005.

[133] J radi M, Riffat S. Modelling and testing of a hybrid solar-biomass ORC-based micro-CHP system. IntJ Energy Res 2014;38:1039-52. doi:10.1002/er.3145.

[134] Liu Q, Bai Z, Wang X, Lei J , J in H. Investigation of thermodynamic performances for two solarbiomass hybrid combined cycle power generation systems. Energy Convers Manag 2016;122:252- 62. doi:10.1016/j.enconman.2016.05.080.

[135] Khan EU, Martin AR. Optimization of hybrid renewable energy polygeneration system with membrane distillation for rural households in Bangladesh. Energy 2015;93:1116- 27. doi:10.1016/j.energy.2015.09.109.

[136] Chow TT. A review on photovoltaic/thermal hybrid solar technology. Appl Energy 2010;87:365- 79. doi:10.1016/j.apenergy.2009.06.037.

[137] Uris M, Linares J I, Arenas E. Size optimization of a biomass-fired cogeneration plant CHP/ CCHP (Combined heat and power/ Combined heat, cooling and power) based on Organic Rankine Cycle for a district network in Spain. Energy 2015;88:935- 45. doi:10.1016/j.energy.2015.07.054.

[138] Hashim H, Ho WS, Lim J S, Macchietto S. Integrated biomass and solar town: Incorporation of load shifting and energy storage. Energy 2014;75:31- 9. doi:10.1016/j.energy.2014.04.086.

[139] Wang \$ R Z, Oliveira RG. Adsorption refrigeration-An efficient way to make good use of waste heat and solar energy. Prog Energy Combust Sci 2006;32:424- 58. doi:10.1016/j.pecs.2006.01.002. 
[140] Deng J, Wang RZ, Han GY. A review of thermally activated cooling technologies for combined cooling, heating and power systems. Prog Energy Combust Sci 2011;37:172- 203. doi:10.1016/j.pecs.2010.05.003.

[141] Daou K, Wang RZ, Xia ZZ. Desiccant cooling air conditioning: a review. Renew Sustain Energy Rev 2006;10:55- 77. doi:10.1016/j.rser.2004.09.010.

[142] Balcombe P, Rigby D, Azapagic A. Investigating the importance of motivations and barriers related to microgeneration uptake in the UK. Appl Energy 2014;130:403- 18. doi:10.1016/j.apenergy.2014.05.047.

[143] Renewable Energy Agency I. IRENA Battery Storage Report 20152015. http:// www.irena.org/ DocumentDownloads/Publications/IRENA_Battery_Storage_report_20 15.pdf (accessed March 7, 2017).

[144] Daghi M, Sedghi M, Ahmadian A, Aliakbar-Golkar M. Factor analysis based optimal storage planning in active distribution network considering different battery technologies. Appl Energy 2016;183:456-69. doi:10.1016/j.apenergy.2016.08.190.

[145] Sun C, Liu J , Gong Y, Wilkinson DP, ZhangJ . Recent advances in all-solid-state rechargeable lithium batteries. Nano Energy 2017;33:363- 86. doi:10.1016/j.nanoen.2017.01.028.

[146] Fang Y, Kuang S, Gao X, Zhang Z. Preparation and characterization of novel nanoencapsulated phase change materials. Energy Convers Manag 2008;49:3704- 7. doi:10.1016/j.enconman.2008.06.027.

[147] Wu D, Chen J , Roskilly AP. Phase change material thermal storage for biofuel preheating in micro trigeneration application: A numerical study. Appl Energy 2015;137:832- 44. doi:10.1016/j.apenergy.2014.09.087.

[148] Khatri KK, Sharma D, Soni SL, Tanwar D. Experimental investigation of CI engine operated Micro-Trigeneration system. vol. 30. 2010. doi:10.1016/j.applthermaleng.2010.02.013.

[149] Clack CTM, Qvist SA, Apt J , Bazilian M, Brandt AR, Caldeira K, et al. Evaluation of a proposal for reliable low-cost grid power with $100 \%$ wind, water, and solar. Proc. Natl. Acad. Sci. U. S. A., National Academy of Sciences; 2017, p. 201610381. doi:10.1073/pnas.1610381114.

[150] An Independent Evaluation of the El Hierro Wind \&amp; Pumped Hydro System | Energy Matters n.d. http:// euanmearns.com/ an-independent-evaluation-of-the-el-hierro-windpumped-hydro-system/ (accessed J une 26, 2017).

[151] Abbas T, Ali G, Ali S, Khalid M, Asif M. Economic analysis of biogas adoption technology by rural farmers: The case of Faisalabad district in Pakistan. Renew Energy 2017;107:431-9. doi:10.1016/j.renene.2017.01.060.

[152] Aslam Bhutta MM, Hayat N, Bashir MH, Khan AR, Ahmad KN, Khan S. CFD applications in various heat exchangers design: A review. Appl Therm Eng 2012;32:1- 12. doi:10.1016/j.applthermaleng.2011.09.001.

[153] Li Q, Flamant G, Yuan X, Neveu P, Luo L. Compact heat exchangers: A review and future applications for a new generation of high temperature solar receivers. Renew Sustain Energy Rev 2011;15:4855- 75. doi:10.1016/j.rser.2011.07.066.

[154] Ahrenfeldt J, Thomsen TP, Henriksen U, Clausen LR. Biomass gasification cogeneration - A review of state of the art technology and near future perspectives. Appl Therm Eng 2013;50:1407- 17. doi:10.1016/j.applthermaleng.2011.12.040.

[155] Susastriawan AAP, Saptoadi H, Purnomo. Small-scale downdraft gasifiers for biomass gasification: A review. Renew Sustain Energy Rev 2017;76:989- 1003. doi:10.1016/j.rser.2017.03.112.

[156] Rong A, Lahdelma R. Role of polygeneration in sustainable energy system development challenges and opportunities from optimization viewpoints. Renew Sustain Energy Rev 2016;53:363- 72. doi:10.1016/j.rser.2015.08.060.

[157] Khan EU, Mainali B, Martin A, Silveira S. Techno-economic analysis of small scale biogas based 
polygeneration systems: Bangladesh case study. Sustain Energy Technol Assessments 2014;7:68- 78. doi:10.1016/j.seta.2014.03.004.

[158] Khan MMA, Saidur R, Al-Sulaiman FA. A review for phase change materials (PCMs) in solar absorption refrigeration systems. Renew Sustain Energy Rev 2017;76:105- 37. doi:10.1016/j.rser.2017.03.070.

[159] Kim JG, Son B, Mukherjee S, Schuppert N, Bates A, Kwon O, et al. A review of lithium and nonlithium based solid state batteries. J Power Sources 2015;282:299- 322. doi:10.1016/j.jpowsour.2015.02.054.

[160] Lai SM, Hui CW. Integration of trigeneration system and thermal storage under demand uncertainties. Appl Energy 2010;87:2868- 80. doi:10.1016/j.apenergy.2009.06.029.

[161] Ameri M, Besharati Z. Optimal design and operation of district heating and cooling networks with CCHP systems in a residential complex. Energy Build 2016;110:135- 48. doi:10.1016/j.enbuild.2015.10.050.

[162] Kyriakarakos G, Dounis AI, Rozakis S, Arvanitis KG, Papadakis G. Polygeneration microgrids: A viable solution in remote areas for supplying power, potable water and hydrogen as transportation fuel. Appl Energy 2011;88:4517-26. doi:10.1016/j.apenergy.2011.05.038.

[163] Cuéllar-Franca RM, Azapagic A. Carbon capture, storage and utilisation technologies: A critical analysis and comparison of their life cycle environmental impacts. J CO2 Util 2015;9:82- 102. doi:10.1016/j.jcou.2014.12.001. 